\title{
Couplage structurel entre systèmes produits- services. Cas de l'approvisionnement en granulats de construction de la Région lle-de-France
}

\author{
Structural coupling between product-service systems. Study of the \\ construction aggregates supply in the lle-de-France region
}

\author{
Clément Morlat ${ }^{1}$, Jean-Marc Douguet ${ }^{2}$ \\ ${ }^{1}$ Chercheur associé au laboratoire Clersé, Université de Lille 1, France, clement.morlat@gmail.com \\ ${ }^{2}$ MCF HDR à I'Université Paris Saclay, UVSQ, jean-marc.douguet@uvsq.fr
}

RÉSUMÉ. Lors du programme ANR «AGREGA », un portail internet contributif ( ePLANETe.Blue ») a été mobilisé. ॥ a permis d'accompagner les acteurs de la filière d'approvisionnement de la Région lle-de-France en granulats de construction nécessaires au Grand-Paris dans une démarche de représentation collective des interactions entre stratégies d'économie circulaire et enjeux de soutenabilité du territoire francilien. Dans une logique d'économie circulaire, la filière francilienne d'approvisionnement en granulats peut être abordée comme un système produitsservices (SPS) orienté-produit. Si l'on s'intéresse plus largement aux enjeux de soutenabilité sociale, environnementale et économique liés à l'inscription de cette filière dans le territoire francilien, un SPS orienté-usage peut être objectivé. Le portail internet " ePLANETe.Blue » permettrait à des acteurs de ce territoire d'aller plus loin en coproduisant une méthodologie d'évaluation ad hoc destinée à favoriser un couplage structurel entre ces deux SPS.

ABSTRACT. During an ANR (French National Research Agency) project called "AGREGA", a contributory Web portal (ePLANETe.Blue) was developed. It supported actors involved in the supply chain of the construction of aggregates, which was needed in the Paris area. This was done in a process of collective compromises between strategies of the circular economy and issues of the supply chain in the lle-de-France region. From a circular economy perspective, this supply chain can be considered as a product-oriented product-service system (PSS). From a more regional perspective regarding social, economic and ecological sustainability issues, the same supply chain could be considered as a use-oriented PSS. The "ePLANETe.Blue" Web portal was designed to help actors and stakeholders get involved in the co-production of an ad hoc evaluation methodology which could facilitate a structural coupling between those two types of PSSs.

MOTS CLEFS. Economie Circulaire, Systèmes Produits-Services, Economie de la Fonctionnalité, Délibération, Web Contributif.

KEYWORDS. circular economy, product-service system, functional economy, deliberation, contributory Web portal.

\section{Introduction}

Ce texte propose une démarche de représentation et d'évaluation des interactions entre une filière industrielle et un territoire. Il se fonde sur les résultats du projet ANR «AGREGA »«Anticipation et Gestion régionales des Ressources En GranulAts» qui s'intéresse à l'approvisionnement de la Région Ile-de-France en granulats nécessaires à la production de béton de construction, et à la gestion des déchets de construction du Grand Paris. Dans une logique d'économie circulaire, améliorer la filière des granulats de construction en Ile-de-France implique de qualifier les performances de cette filière en termes de flux de matière. Elargir le domaine de performances considérées peut amener à étudier comment la circularité de l'économie des granulats s'inscrit dans les grands cycles de la biosphère (carbone, eau, etc.). Il est possible d'élargir encore l'analyse des performances en s'intéressant aux effets sociaux et économiques engendrés par cette filière à différentes échelles de territoire. 
Une deuxième partie mobilise le concept de système produit-service (SPS) pour inscrire la dimension industrielle de la filière des granulats au sein d'une question sociale portant sur des enjeux de développement territorial soutenable. Deux SPS peuvent être considérés. L'un décrivant la manière dont la circularisation de l'économie des granulats engendre des avantages fonctionnels qui peuvent être reconnus comme des services par les acteurs industriels de la filière. L'autre décrivant la manière dont cette circularisation améliore la soutenabilité de l'inscription de la filière dans le territoire: les avantages sociaux, économiques et écologiques du changement de conformation de la filière peuvent être reconnus comme des services par les acteurs du territoire.

Si ensuite l'interaction entre ces deux SPS est reconnue, c'est à dire si d'une part les acteurs du territoire reconnaissent l'intérêt qu'il y aurait à favoriser la circularisation de la filière, et que d'autre part les acteurs de la filière reconnaissent l'intérêt qu'il y aurait à améliorer la soutenabilité sociale, économique et écologique de leur activité industrielle, un couplage fonctionnel peut être représenté. Et si une évolution du processus de gouvernance territoriale intègre la reconnaissance partagée de ces services réciproques et amène les acteurs industriels à ré-envisager leurs performances individuelles au regard du fonctionnement d'ensemble de la filière, et à reconfigurer en conséquence la structure de cette filière, le couplage fonctionnel s'incarne structurellement dans la configuration des modèles d'affaires et des flux de matières. On peut alors aussi parler de couplage structurel.

Une troisième partie s'intéressera aux modes de représentation d'un SPS sur le portail web «ePLANETe.Blue ». Il s'agit notamment de resituer des indicateurs mobilisés pour la représentation et la reconnaissance de tels avantages réciproques, par rapport à un contexte particulier caractérisé par des acteurs, des enjeux, des scénarios et des connaissances disponibles.

Une démarche de coproduction d'un dispositif d'évaluation normative, qualitative et quantitative, des performances d'un SPS sera ensuite proposée, fondée sur le principe de l'analyse multi-acteurs et multicritères.

Le portail «ePLANETe.Blue » permet ainsi d'accompagner la construction de la signification sociale d'un ou de plusieurs SPS, et de leurs interactions. A l'aide d'une matrice de délibération, les acteurs peuvent questionner et faire évoluer ensemble les connaissances utilisées pour représenter un SPS et ses performances, et en augmenter ainsi les ambitions.

\section{Enjeux liés à la circularisation de l'économie des granulats de construction en lle de France}

\subsection{Cas d'étude : projet ANR AGREGA}

Avec la construction du «Grand Paris Express », la construction de 70000 logements par an, l'organisation des jeux olympiques en 2024, le projet «Europacity », et d'autres encore, l'approvisionnement de la Région Ile-de-France en granulats de construction - constituant principal des bétons - est devenu un enjeu majeur pour les décennies qui viennent [PAN 17]. A ces contraintes, de fait, s'ajoutent des contraintes environnementales (préconisation par la DRIEE d'une diminution de la production des granulats alluvionnaires, ressource la plus prisée) et des contraintes sociétales (résistance des riverains) [PIP 16]. Le recours au recyclage, qui constitue une option stratégique bénéficiant d'une meilleure acceptabilité par les riverains (possibilité d'implantation en zone urbaine, remblaiement de carrières), reste toutefois limité (ratio plafonné dans la fabrication des bétons, coût de manutention jugé élevé, concurrence des installations de stockage de déchets ultimes ISDI, etc.). 
De nombreux outils (modèles) d'évaluation objective des systèmes "granulats/déchets BTP" sont disponibles. Ces outils ne permettent cependant pas d'aborder les phénomènes socioéconomiques décrits ci-dessus. Une capacité des parties prenantes à dialoguer afin co-construire des scénarios contribuerait à la mise en œuvre des futurs schémas régionaux de carrières et plan régional de gestion des déchets BTP. Mobiliser des moyens scientifiques visant à reconnaitre publiquement la pluralité des valeurs permettrait aux acteurs de se forger davantage d'opinions, parfois diverses, sur ces scénarios. Par ailleurs, cela permettrait aussi d'admettre les contradictions existantes pour découvrir des manières originales de les articuler, favoriserait l'action [CHA 09].

Dans le cadre d'un programme de recherche ANR «Vers une économie circulaire méthodologie et services associés », le projet «AGREGA», achevé en début d'année 2018, a contribué à la fourniture de ces moyens scientifiques de dialogue, via le développement de trois outils qui sont indépendants dans leur utilisation, mais complémentaires (voir figure 1). La finalité de la démarche était que les scénarios coproduits puissent être simulés via un modèle de représentation « objective» (estimation de variables), évalués par représentation «subjective » fondée sur des jugements d'acteurs (signification sociale), et interprétés collectivement par un jeu de rôles associant estimation et construction du sens.

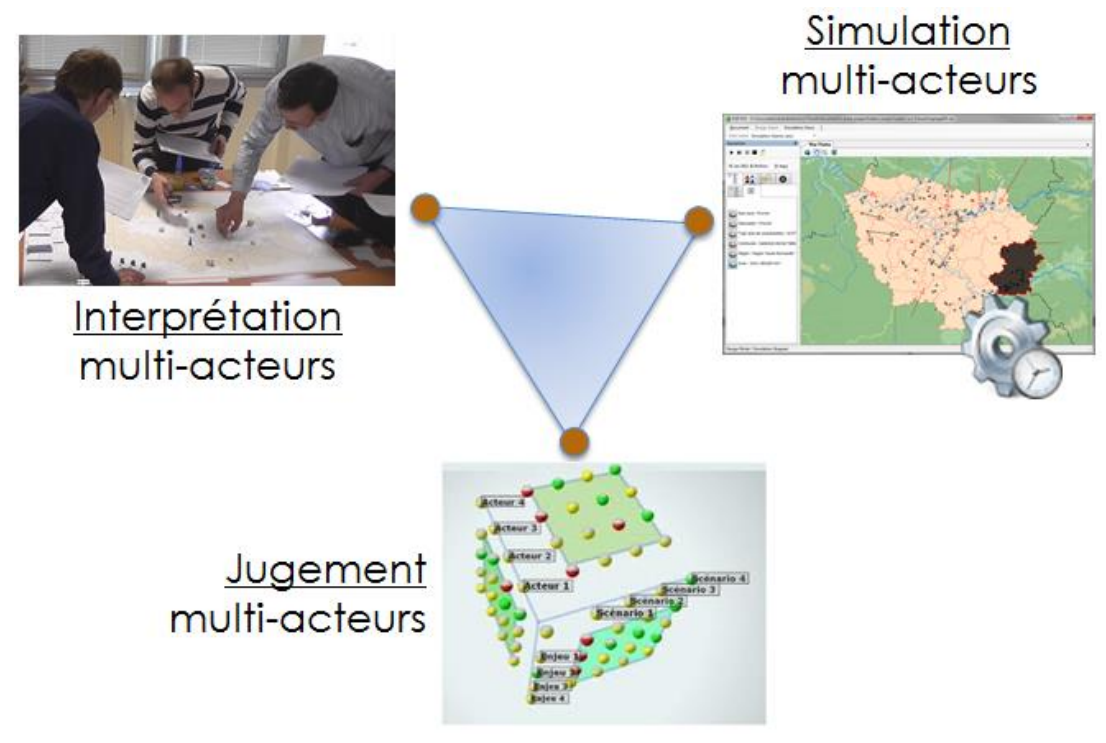

Figure 1. Les trois outils développés dans le cadre d'AGREGA

Le projet AGREGA a permis de rapporter, d'analyser et de discuter (sur le plan thématique et méthodologique) une première réalisation de ce processus d'évaluation avec les différents acteurs de la Région Ile-de-France qu'il a été possible de rencontrer [DOU 18]. Le défi de cette évaluation tenait à la manière d'intégrer la question de la circularité de l'économie du secteur des granulats.

\subsection{Economie circulaire des granulats et grands cycles de la biosphère}

L'économie circulaire désigne un modèle économique dont l'objectif est de produire des biens et des services de manière soutenable, en limitant la consommation et les gaspillages de ressources (matières premières, eau, énergie) ainsi que la production des déchets. Il s'agit de rompre avec le modèle de l'économie linéaire (extraire, fabriquer, consommer, jeter) pour aller vers un modèle économique «circulaire», décrit par la règle des « $4 \mathrm{R} »$ (Réduire, Réemployer, Réutiliser, Recycler). Ce modèle d'économie circulaire permet de réduire le flux de matières et d'énergie pour un même niveau de réponse aux besoins, donc contribue à un découplage entre réponse aux besoins par l'économie, et impacts négatifs sur les écosystèmes. 
C'est un enjeu majeur pour la filière des granulats [PIP 16]. La règle des «4R » peut être déclinée selon quatre objectifs clefs : réduire, réemployer, réutiliser, recycler.

Dans le contexte de la filière des granulats, la réduction porte sur l'exploitation des alluvionnaires (pour préserver l'environnement), au profit de granulats à moindres enjeux environnementaux (roches massives), ainsi que sur la mise en décharge des déchets, au profit du recyclage ou de la valorisation. Le réemploi à favoriser est celui des déblais de terres excavées issues de la déconstruction, pour les remblaiements sur le même chantier, ou ailleurs; La réutilisation à promouvoir est celle des déchets inertes de bétons et d'enrobés issus de la déconstruction (pour les constructions de route et pour le remblaiement des carrières en fin de vie); Le recyclage concerne aussi les déchets qui ne peuvent pas être réutilisés directement, et leur intégration dans la construction des routes.

La figure 2 propose une expression des formes de circularité du secteur des granulats, en termes de matières. Cette figure est inspirée de [PAN 17] mais la partie « déchets » se focalise ici sur les déchets inertes (DI) du BTP.

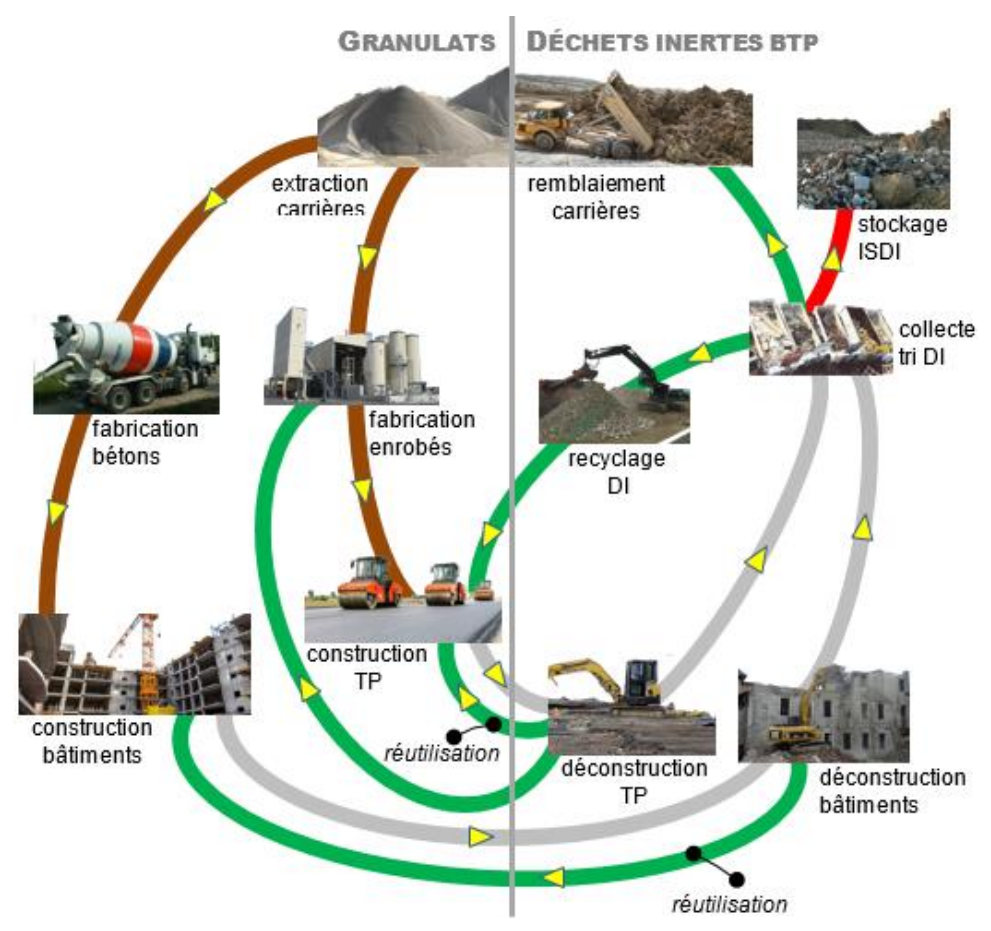

Figure 2. Une vue de la circularité de la filière " granulats ", focalisée, pour le circuit secondaire, sur les déchets inertes (DI) du BTP

Dans le reste de cet article, sauf mention contraire, le terme « déchets » fera implicitement référence aux déchets inertes du BTP.

Réunir en boucle les processus économiques de la filière des granulats, c'est à dire circulariser l'économie des granulats, entraine une réduction de la consommation de ressources naturelles (dont l'énergie) et des émissions nettes de déchets. Les éco-innovations - techniques ou sociales - qui favorisent ces boucles vertueuses, contribuent donc au maintien des Grands Cycles de la biosphère.

\subsection{Le défi de l'évaluation des performances d'un système complexe}

L'étude socioéconomique menée en initiation du projet AGREGA identifiait onze enjeux de soutenabilité de la filière francilienne d'approvisionnement en granulats [CHA 15]. Leur compréhension a été approfondie lors du déroulement de ce projet [DOU 18]. 
Répondre à la demande en granulats constitue un défi. L'Ile-de-France en consomme environ 30 millions de tonnes chaque année, essentiellement pour le béton nécessaire aux logements et aux futures infrastructures de transport. Cette demande n'est pas homogène sur le plan géographique. De plus, le Grand Paris entraine une inconnue importante concernant l'importance des besoins futurs. Pour certains acteurs, il y a des risques de tensions sur les matériaux. Un manque d'information sur les besoins rend difficile le travail de planification collective. Ce défi est d'autant plus sensible que les acteurs de la filière doivent s'efforcer de maintenir le taux d'indépendance de la Région. L'Ilede-France extrait environ 10 millions de tonnes de granulats par an, auxquels il faut ajouter une production de matériaux alternatifs issus du recyclage des bétons, des enrobés et des mâchefers d'incinération d'ordures ménagères (5 millions de tonnes). Avec sa consommation annuelle, la région est déficitaire à hauteur de $45 \%$ en moyenne et doit importer des matériaux des régions proches (mais aussi moins proches, notamment de Belgique). Cet export depuis les autres régions vers la région francilienne entraine parfois un sentiment de surexploitation de la part des régions concernées, d'autant que l'activité n'est alors pour elles pas tellement créatrice d'emplois, les matériaux étant transformés en Ile-de-France. Les décisions d'export vers l'Ile-de-France relèvent de choix politiques.

Il convient, de plus, de limiter la contribution de la filière aux changements climatiques et à la pollution. Les activités de la filière des granulats consomment de l'énergie et rejettent des gaz à effet de serre et des particules pour l'extraction, la transformation et surtout le transport des ressources. L'impact varie selon les matériaux et les modes de transport. Cependant, les difficultés d'accès à la ressource entrainent un éloignement progressif des sites de production et donc un allongement des distances à parcourir. La protection de la ressource en eau est un autre enjeu à considérer. L'exploitation de granulats peut engendrer des impacts sur cette ressource : consommations d'eau pour le lavage et la transformation, risque de la proximité de champs captant, etc. L'exploitation des granulats alluvionnaires, qui sont les plus prisés, pose particulièrement problème. Par ailleurs, la question de savoir si les efforts de réaménagement post exploitation peuvent amener à considérer que les activités de carrières sont favorables à une préservation des milieux naturels soulève des points de vue différents. Cependant, les impacts de l'activité sur les milieux naturels existent. L'accroissement de l'intérêt pour la préservation de l'environnement au sein de la société entraîne un accès de plus en plus ardu à la ressource avec une augmentation croissante des zones d'exclusion.

Par ailleurs, même si les réserves de granulats sont, dans l'ensemble, encore importantes en France, il s'agit d'une ressource épuisable à préserver. Une raréfaction des alluvionnaires, matériau de grande qualité désormais réservé aux usages nobles comme la construction (alors qu'il était auparavant utilisé aussi pour les routes) implique d'envisager des alternatives auxquelles pourrait contribuer le recyclage. Maintenir les emplois est un autre enjeu évoqué. Les carrières sont pourvoyeuses d'emplois, majoritairement peu qualifiés et non-délocalisables. Les 1550 entreprises franciliennes emploient ainsi 14660 salariés. Les emplois indirects sont estimés à 50000 [UNI 15]. La hausse des besoins conduit cependant à l'épuisement des stocks et à un fort recours aux importations, ce qui fragilise l'emploi dans les carrières franciliennes. Le recyclage ralentirait cette hausse des besoins et contribuerait aussi à créer de l'emploi non délocalisable. Les acteurs de la filière ont cependant des difficultés à assurer la rentabilité économique de leur activité sur le long terme. Or, la visibilité concernant le niveau de recyclage local des matériaux de déconstruction issus du BTP est stratégique. Elle permet d'envisager les modalités de réponse à la demande régionale et contribue aussi à nourrir les réflexions concernant les investissements fonciers lourds que doivent assumer les entreprises exploitantes de carrières. Leur modèle économique est caractérisé par une inertie qui les empêche de changer de stratégie rapidement (des demandes d'ouverture de carrières peuvent prendre plus de 10 ans). 
Il convient d'autre part de livrer la ressource là où se trouve le besoin. Le granulat est une ressource pondéreuse mais peu chère, la part du transport dans son prix final est donc importante : ce prix double tous les $50 \mathrm{kms}$. Une massification du transport de la ressource améliorerait la rentabilité économique. Elle impliquerait de tenir compte de la configuration de la région Ile-deFrance et des problèmes d'acheminement (trafic routier, difficultés de traverser la capitale par la Seine) - l'approvisionnement devant se faire de tous les côtés. De plus, une grande partie de la consommation se fait en zone dense, là où le transport est déjà saturé. On note également une différence entre les lieux de consommation et de production: la petite couronne concentre une grande part de la consommation mais ne compte aucun site de production (excepté le recyclage). Limiter les nuisances pour les riverains est alors un enjeu à considérer. Les granulats ne profitent pas, ou peu, aux habitants qui doivent pourtant subir des nuisances: bruit, poussières, trafic, modification du paysage, etc. L'expression du syndrome Nimby est forte vis à vis de beaucoup d'activités industrielles, et plus particulièrement face à l'ouverture des carrières. Les élus, peu sensibilisés à la question des matériaux, suivent souvent l'avis de leurs concitoyens en s'opposant aux projets (notamment via les règlements d'urbanisme). Assurer la qualité et sécurité des ouvrages en béton est un autre enjeu incontournable. Il entre cependant en concurrence avec l'enjeu de préservation de la ressource granulat. Une préférence pour les granulats vierges - ou "naturels" reste en effet fortement ancrée, même lorsqu'elle ne correspond pas à une exigence technique, normative ou réglementaire. Des travaux de $R \& D$ sont réalisés au niveau des différents métiers (constructeurs, fabricant de béton et de ciment, carriers) afin d'assurer la même qualité d'ouvrage avec moins de matériaux nobles (alluvionnaires) ou vierges, et d'accompagner le développement de nouvelles techniques liées au recyclage.

La circularisation de l'économie des granulats, et son impact positif sur les cycles de la biosphère, sont inscrits dans un système complexe dont l'évaluation implique l'analyse intégrée de multiples dimensions de soutenabilité. Pour les aborder, nous avons appliqué la démarche INTEGRAAL [OCO 11].

\subsection{La méta-méthode INTEGRAAL pour structurer l'évaluation des performances}

La démarche INTEGRAAL vise à engager experts et parties prenantes dans un processus d'évaluation intégrée, à travers 6 étapes principales (dont la réalisation n'est pas forcément linéaire, comme l'illustre la figure 3).

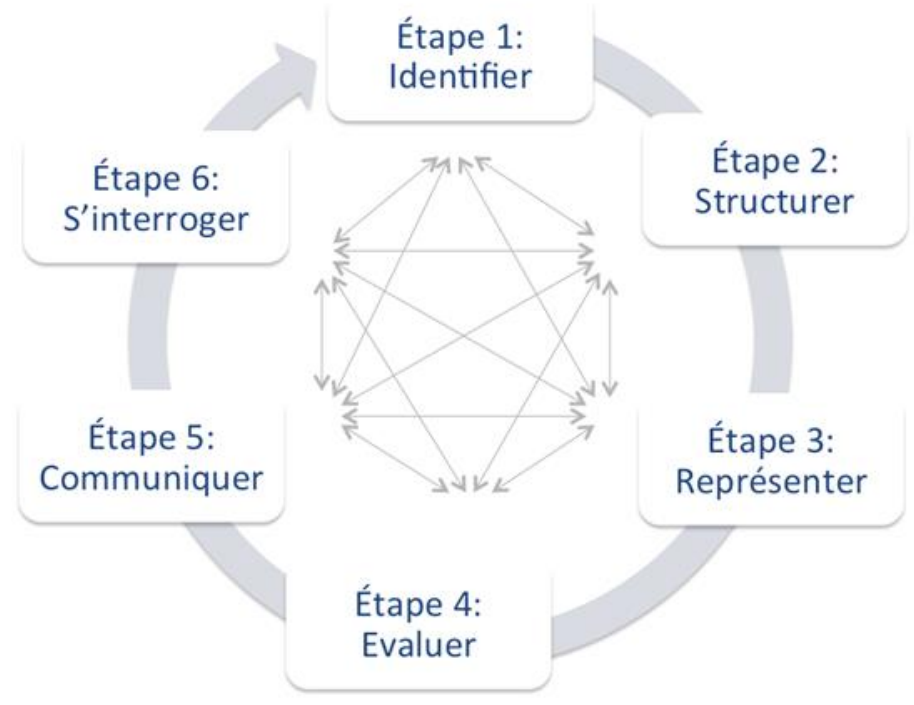

Figure 3. Etapes de la méta-méthode INTEGRAAL ${ }^{T M}$. Adapté depuis (O'Connor et Da Cunha ; 2011) 
Etape 1 : Identification des terrains, des objectifs et des scénarios d'extraction des granulats et/ou de recyclage des bétons (échelles régionale et locale).

Etape 2 : Structuration du problème de choix social, en termes de parties prenantes, de catégories d'enjeux de performance (ici les onze enjeux de soutenabilité décrits au point précédent) et d'options d'action. Cette étape repose sur une étude approfondie du terrain (dont des entretiens) et de la littérature.

Etape 3 : Mobilisation d'outils pour représenter la situation afin de déterminer des systèmes d'indicateurs évolutifs qui caractériseront les formes de circularité, les performances de soutenabilité de l'ancrage territorial de la filière, ainsi que les zones et les territoires aux échelles régionales et locales.

Etape 4 : Mobilisation des acteurs de l'étape 2 pour une évaluation multicritère des scénarios de circularisation de l'économie des granulats, à partir d'une gamme plus ou moins large des indicateurs obtenus précédemment et qui seront transférés vers les outils de l'étape 3 grâce auxquels ils seront analysés.

Etape 5 : Communication des résultats de l'étude auprès des participants mais aussi du public de façon plus large. On passe ici de la recherche au processus de décision.

Etape 6 : Retours sur expériences sur le déroulement de la démarche d'évaluation.

\section{Encadré 1. Déclinaison de la démarche INTEGRAAL au cas particulier de la filière des granulats [DOU 18].}

\section{Retenir l'approche Système Produit-Service}

Chaque étape d'INTEGRAAL a été déroulée entièrement lors du projet AGREGA. Nous décrivons dans cet article la possibilité d'ajouter à l'étape 3 une nouvelle forme de représentation, inspirée du concept de Système Produit-Service, et à l'étape 4 un outil d'évaluation des performances de soutenabilité du système régional d'approvisionnement en granulats.

\subsection{Approche et typologie des systèmes produits-services}

Le concept de Système Produit-Service (SPS) fait référence à un système de produits, de services, de réseaux d'acteurs et d'infrastructures orienté vers une quête continue de compétitivité, de satisfaction des besoins clients, et dans l'ambition d'un impact environnemental plus faible que les modèles économiques traditionnels [GOE 99]. L'utilisation de ce concept s'inscrit dans le champ d'une Economie de la Fonctionnalité dont l'ambition est de créer la plus haute valeur d'usage possible, le plus longtemps possible, et cela en consommant aussi peu de ressources matérielles et d'énergie que possible [STA 86]. Dans une économie de la fonctionnalité, le bien physique est considéré comme une immobilisation et non plus comme un consommable [LAU 08]. La dimension physique de l'appareil productif, c'est à dire l'économie industrielle, devient donc un support de la création de valeur, et non plus son processus central. C'est un changement de paradigme : l'essentiel de la productivité est déterminé par la mise en disponibilité d'un potentiel de phénomènes fonctionnels qui sont qualifiés de fonctionnalités lorsqu'un acteur en reconnaît une utilité. Et l'essentiel de la rentabilité est déterminé par la capacité à accorder un prix à ces utilités dans le cadre d'un contrat de service. Les SPS constituent un formalisme utile à cette économie.

Une distinction peut être opérée entre trois types de SPS [HOC 02] [LAP 13].

- SPS orienté résultat : considérée comme la plus innovante, cette stratégie correspond à la mise à disposition sobre en énergie et matière d'un phénomène fonctionnel généré spécifiquement, et dont les coûts sont directement assumés par les utilisateurs.

Ici la propriété du bien physique n'est pas transférée. Ce bien n'est qu'un support mobilisé pour produire des fonctionnalités et les mettre à disposition de l'utilisateur. C'est l'utilité de ces fonctionnalités qui est l'objet de la transaction. 
- SPS orienté usage : cette stratégie correspond à la mise à disposition, sans transfert de propriété, de l'usage d'un ensemble de biens matériels dont l'articulation par l'utilisateur génère des phénomènes fonctionnels valorisables.

Les biens physiques occupent une place plus centrale : ils préexistent à l'expression d'un besoin de fonctionnalités. C'est cependant toujours l'agencement fonctionnel de ces biens qui est l'objet de la transaction, non pas leur commercialisation.

- SPS orienté produit : c'est la stratégie la moins innovante, elle correspond à une prestation de service tournée vers l'augmentation de la fonctionnalité ou de la longévité de produits après leur acquisition par l'utilisateur.

Cette dernière option relèverait plutôt d'une "version durable" du service après-vente traditionnel que d'une économie de la fonctionnalité, du fait du transfert de propriété du bien physique [LAP 13].

Comme l'illustre la figure 4, ces trois stratégies balisent un gradient d'innovation dans lequel une multitude de nuances possibles peuvent être envisagées, depuis des valorisations principalement portées sur le produit physique et jusqu'à des valorisations principalement fondées sur le service.

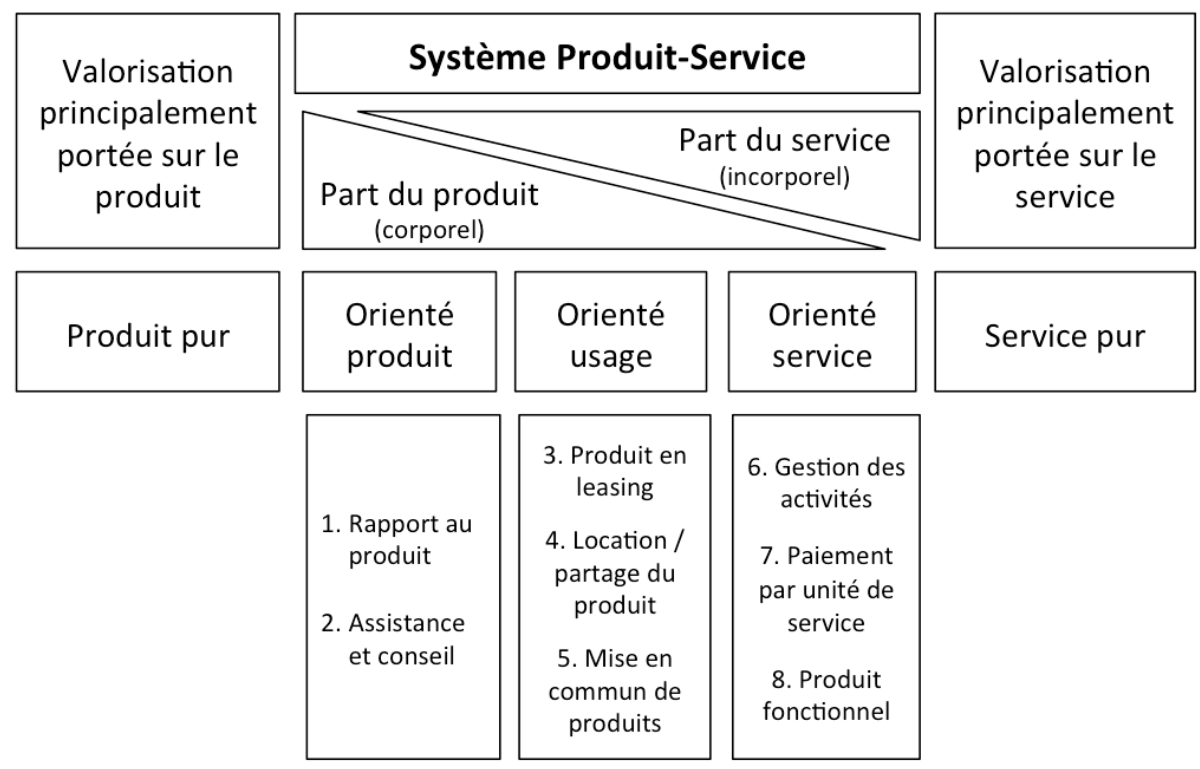

Figure 4. Catégories principales et subsidiaires de SPS. Adapté et traduit depuis [TUK 04].

L'approche SPS nous intéresse tout d'abord en ce qu'elle permet d'aborder la circularisation d'une économie du point de vue du découplage entre impacts écologiques et valorisation économique. En effet, l'économie circulaire ne fonde pas la valorisation sur le transfert de propriété du bien physique, ce qui irait à l'encontre de l'ambition de réduction des flux de matières et d'énergie. Ainsi, les stratégies SPS les plus ambitieuses invitent à envisager l'économie circulaire dans une approche socioéconomique. Plus l'ambition d'innovation SPS augmente, plus la part de l'innovation socio-économique dans la création de valeur prend le pas sur celle de l'innovation technico-économique. En effet, plus la capacité territoriale de valorisation des fonctionnalités d'un même ensemble de produits physiques augmente, moins le recours à d'autres productions industrielles est nécessaire, ce qui permet de limiter le flux énergie-matières.

Un SPS est avant tout une construction sociale [MOR 06]. C'est le lien tissé au sein d'un réseau d'acteurs qui préfigure la relation entre sobriété énergie-matières d'une solution de production, fonctionnalités multiples générées, contractualisation de la mise à disposition des avantages et équilibre économique d'un SPS. Ce lien peut s'apprécier au niveau d'une entité du territoire, en considérant son impact sur d'autres entités de ce territoire. Les connaissances d'un concepteur- 
producteur peuvent ainsi être envisagées comme le fondement d'une offre intégrée qui permet d'augmenter la valeur de sortie d'un SPS, tout en diminuant les coûts des matières et autres flux à son entrée [BAI 07]. Le lien entre acteurs peut aussi, conjointement, être objectivé à l'échelle du territoire. Lors des processus d'innovation qui caractérisent l'économie de la fonctionnalité, les interdépendances entre acteurs engendrent leur coévolution. Une spécialisation concertée (coconception, coproduction et coévaluation) les inscrit dans une logique de programmation collective visant à démultiplier les fonctionnalités valorisables tout en s'assurant de leur complémentarité [VAI 09].

Dans le contexte de la filière des granulats, les stratégies SPS peuvent donc être envisagées d'une part à l'échelle du lien entre les activités industrielles du territoire, dans une logique de circularisation des flux de matières et d'énergie, et d'autre part à l'échelle du lien entre parties prenantes du territoire et acteurs industriels de la filière, considérant que la circularisation de cette filière rend service au territoire. L'objectif de programmation collective est donc double : d'une part, amener les acteurs de la filière industrielle à augmenter au maximum la longévité de l'usage du flux de granulats; d'autre part amener les parties prenantes du territoire à reconnaître dans cette augmentation un maximum de fonctionnalités utiles (notamment en termes de réponse aux onze enjeux de soutenabilité décrits point 1.3.).

\subsection{Diversité des échelles et des regards sur les performances}

Les trois stratégies SPS évoquées au point précédent peuvent être articulées pour aborder la diversité des enjeux et des échelles d'analyse du système d'approvisionnement des granulats en Ilede-France.

\subsubsection{Echelle du réseau d'industries et regard porté sur les flux de matières}

La vente de granulat recyclé/réutilisé au sein du système d'activités industrielles qui constitue la filière des granulats s'accompagne d'un service en termes d'extension de la durée de vie des granulats mobilisés (longévité du produit à l'échelle de la filière) et en termes de diminution de consommation de ressources vierges (fonctionnalité à l'échelle de la filière). Le système associé à cette économie peut être représenté comme un SPS orienté produit.

\subsubsection{Echelle régionale et regard porté sur la soutenabilité de la filière des granulats}

La présentation succincte des 11 enjeux déterminés dans le cadre du projet AGREGA indique qu'une amplification du recyclage au sein de la filière des granulats serait source de fonctionnalités utiles au territoire francilien. La vente de granulats recyclés au sein d'un système sociotechnique incluant les acteurs industriels de la filière et les acteurs du territoire s'accompagne donc de phénomènes fonctionnels distincts du transfert de propriété des granulats. Le système associé à la soutenabilité de l'ancrage territorial de la filière des granulats peut être représenté comme un SPS orienté usage.

\subsubsection{Regards multi-scalaires portés sur les interactions entre circularité et soutenabilité}

Les enseignements du projet AGREGA [DOU 18] mettent en évidence des interactions fonctionnelles entre les deux SPS décrits précédemment. De façon non exhaustive, ces interactions peuvent être illustrées ainsi :

- Une augmentation du recyclage à l'échelle des constructions franciliennes (performance de circularité de la matière) contribuerait à fixer l'emploi local tout en limitant l'épuisement des carrières régionales, ce qui permettrait notamment de réduire les volumes et distances d'imports donc les coûts et émissions associés (performances de soutenabilité de l'ancrage territorial de la filière). 
- Une capacité de la filière régionale à adopter une conformation permettant de limiter l'expansion de la zone d'approvisionnent (performance de soutenabilité de l'ancrage territorial de la filière), permettrait d'éviter l'ouverture de nouveaux canaux d'approvisionnement longues distances susceptibles de déverser sur le marché local des ressources surabondantes dont la présence casserait l'avantage concurrentiel léger dont bénéficie actuellement le granulat recyclé (performance de circularité de la matière).

Plus généralement, la question du prix du granulat recyclé s'intègre dans un système de performances micro-méso intégré dont la reconnaissance collective permettrait d'élargir l'échelle de conception des équilibres économiques. Si les acteurs du territoire et les acteurs de la filière coconstruisent des scénarios visant à augmenter le recours au recyclage afin de générer spécifiquement les phénomènes fonctionnels qu'ils attendent de ce recyclage, ils constituent ensemble un SPS orienté résultat.

La réalisation d'une offre intégrée micro-mésoéconomique, résultant d'un couplage entre deux SPS - l'un "orienté produit", l'autre "orienté usage" - et donnant lieu à la reconnaissance d'un troisième "orienté résultat", est représentée figure 5.

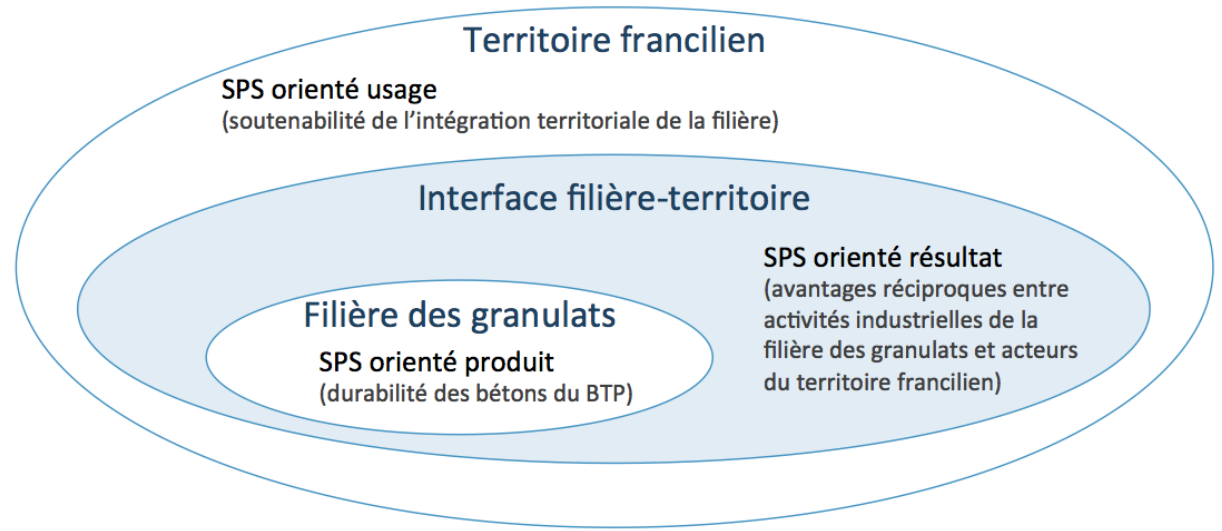

Figure 5. Un SPS orienté résultat, fruit de la reconnaissance des interactions entre un SPS orienté produit et un SPS orienté usage.

La notion de couplage structurel est ici une image didactique, d'inspiration cognitiviste, qui vise à suggérer une analogie analytique avec la notion d'autopoïèse [MAT 94] qui décrit la manière dont un système va se transformer - à partir de sa propre organisation -, pour compenser des perturbations occasionnées par des événements qui lui sont extérieurs. Le fait que ce système s'adapte en continu à ces perturbations en conservant son identité est appelé couplage structurel [MAT 94].

En conscience des limites de l'analogie, nous décrivons ici le fait que la structure méso d'un SPS "orienté-usage" constitué à l'échelle de la filière industrielle des granulats subit les perturbations induites par l'évolution d'une multitude de structures micro des SPS "orienté-produit" constitués autour des activités industrielles qui en sont constitutives. Qualifier d'"avantages fonctionnels" ces perturbations relève d'un processus de reconnaissance sociale consistant à changer de niveau de représentation, afin d'aborder la rationalité économique et politique à l'échelle d'un SPS nouveau: en englobant les deux SPS, "orienté-produit" et "orienté-usage" dans un troisième "orienté-résultat" faisant l'objet d'une programmation collective ad hoc. Il s'agit ainsi d'envisager une nouvelle manière de penser les relations entre le microéconomique et le macroéconomique. 


\subsection{Acteurs industriels du cycle de vie des granulats}

Amener les acteurs d'un SPS orienté-produit et d'un SPS orienté-usage à reconnaître des fonctionnalités utiles à leurs dynamiques réciproques constitue un défi. Une première étape a consisté à organiser le jeu d'acteurs de la dimension industrielle de la filière en suivant la logique de circularisation de la matière.

En complément des développements officiels liés au projet AGREGA, nous avons alors distingué quatre phases d'un « cycle de vie du granulat » (Exploitation, Transports, Utilisation, Recyclage) et identifié des activités impliquées dans chacune de ces phases, en utilisant la nomenclature française NAF Rév.2.1 [INS 15]. Cette liste, destinée à être enrichie, a posé un premier cadre de représentation du cycle de vie des granulats, fondé sur une lecture institutionnelle car basée sur l'identification des acteurs industriels directement impliqués.

Etablir une communication entre ces acteurs et ceux qui sont susceptibles, à d'autres échelles de temps et de territoire, de reconnaître les fonctionnalités utiles d'une inscription soutenable de la filière des granulats dans le territoire francilien constitue un autre défi, que nous avons abordé avec le support du portail web contributif ePLANETe.Blue.

\section{4. ePLANETe.Blue : représentation et analyse de la signification sociale}

La médiation des connaissances entre acteurs impliqués dans plusieurs SPS est compliquée par la variabilité des profils, la multiplicité des points de vue, la diversité des interprétations, des dynamiques d'apprentissage, ainsi que des conflits liés à la mobilisation de règles lors de l'établissement de chemins de décision.

Le portail «ePLANETe.Blue » constitue un dispositif participatif et contributif de modélisation dédiée au choix collectif, constitué notamment de l'ensemble d'outils mobilisés par la méthodologie INTEGRAAL. Une navigation heuristique motivée par liens croisés (Crosslinks) à travers une méta organisation par portes thématiques (voir figure 6), espaces, galeries, profils d'objets, permet d'engager des protagonistes dans une démarche d'amélioration sociale, économique et écologique.

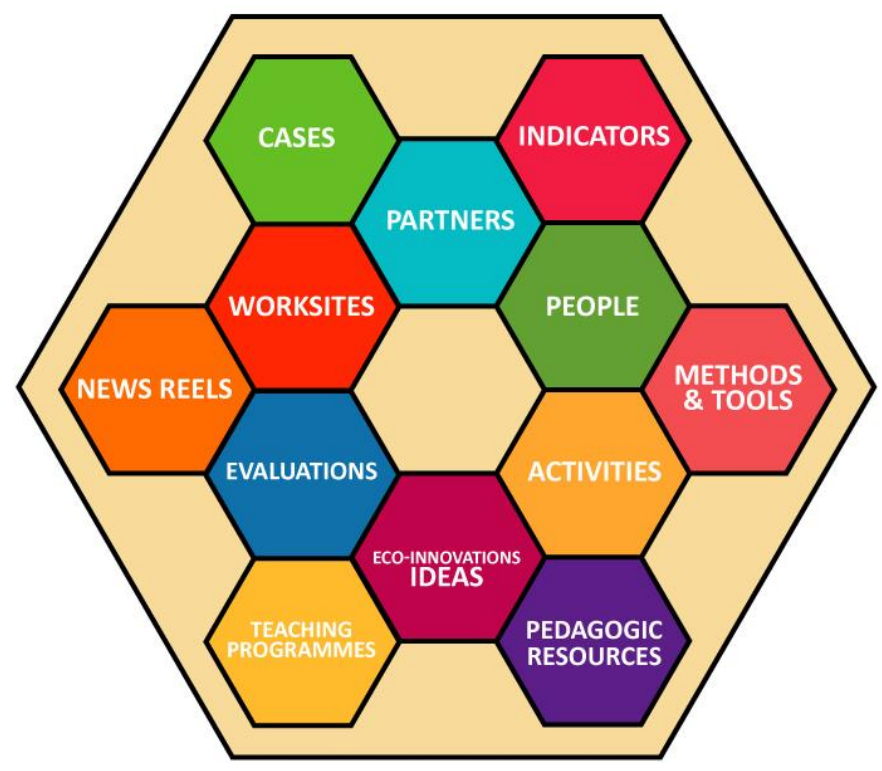

Figure 6. Représentation simplifiée des galeries du portail de connaissances ePLANETe. 
Certaines galeries regroupent des familles d'objets, caractérisées par leurs typologies, pour une création contributive de profils. D'autres, plus complexes, sont enrichies de modes de navigation ou de fonctionnalités propres.

Chaque galerie peut être utilisée de façon spécifique et indépendante. C'est cependant leur utilisation discursive, par navigation de l'une à l'autre, qui renforce la production de connaissances par individuation collective, et favorise un dialogue multi-acteurs.

\section{Représenter un Système Produits-Services}

Les étapes 1 et 2 de la démarche INTEGRAAL (identifier et structurer le problème) ont été décrites dans la partie précédente. L'étape 3 vise à informer et à représenter le système dans lequel s'inscrit le problème étudié. Il s'agit d'identifier les différentes formes de connaissances mobilisables.

\subsection{Indicateurs et connaissances mobilisables}

Des propositions d'indicateurs sont effectuées ex-ante, avant la démarche d'évaluation. Elles serviront d'arguments aux acteurs impliqués dans l'évaluation, lorsque ceux-ci auront à établir un jugement pour caractériser chacun des scénarios co-produits. D'autres indicateurs seront identifiés et mobilisés au cours de la démarche d'évaluation.

Par "indicateur", nous entendons ici toute forme de connaissance, qu'elle ait un caractère quantitatif, qualitatif, en unités de mesure variées (monétaire, physique, etc.). Il est important également de distinguer dans les démarches d'évaluation celles qui visent à établir une estimation, de manière objective, de celles qui permettent d'établir un jugement subjectif. Le caractère attendu des indicateurs varie aussi. On peut distinguer les indicateurs dont on comprend facilement le sens (mais dont les modalités d'estimation sont à préciser), des indicateurs qui sont mesurables (mais dont il faut déterminer le sens) [FRA 11].

Dans le cas qui nous intéresse, différentes sources de connaissances utiles à la construction d'indicateurs peuvent être identifiées a priori : littérature scientifique ; rapports et publications émis par les acteurs liés au projet AGREGA, entretiens réalisés, sessions d'utilisation du jeu de rôle, Systèmes Multi-Agents et modélisation de simulation d'AGREGA, etc.

\subsection{Enrichir les indicateurs par des méta-informations}

La galerie «KIK » (Kiosque aux Indicateurs Kerbabel) du portail «ePLANETe.Blue » présente un catalogue des indicateurs utilisés par chaque communauté d'utilisateurs (voir figure 7). Les indicateurs y sont décrits par des méta-informations : nom, acronyme, description courte, profil scientifique, mode d'interprétation, sources de production (notamment connaissances mobilisées), statut, robustesse, etc.

Un KIK permet de naviguer entre différents indicateurs à partir de filtres thématiques, propose d'établir des liens avec des outils, des terrains ou démarches qui mobilisent ces indicateurs, et offre des accès à des informations pertinentes (documents, sites internet). En plus d'une documentation des indicateurs, il s'agit donc de répertorier les formes de connaissances mobilisées dans le cadre d'un projet, afin de fournir une certaine visibilité et transparence concernant ces connaissances.

Cette galerie peut regrouper l'ensemble des indicateurs retenus, de manière ex-ante ou ex-post, pour la construction des différents outils et démarches de représentation et d'évaluation développées dans le cadre du projet AGREGA. 


\begin{tabular}{ll} 
FILTERS & \\
OPEN CLOSE & $>$ \\
\hline PEOPLE & $>$ \\
PLANET & $>$ \\
PROCESS & $>$ \\
PROFIT & $>$ \\
PROPAGATION & $>$ \\
DIMENSIONS OF SYSTEM ORGANISATION & $>$ \\
ENVIRONMENTAL SERVICES & $>$ \\
ENVIRONMENTAL ASSETS & $>$ \\
ECOSYSTEM TYPE & $>$ \\
SECTORS OF ECONOMIC ACTIVITIES & $>$ \\
UNITS OF ACCOUNTING &
\end{tabular}

View Edit Crosslinks Devel

\section{Livraison des granulats}

Submitted by eplanete on Mon, 07/17/2017 - 18:43

KIK: KIK AGREGA

INFORMATION SOURCE AND STATUS

Source Creation:

Manually by user from Deliberation Matrix (Ex-Post)

KNOWLEDGE QUALITY ASSESSMENT

NUSAP Profile:

0

Community:

AGREGA

Figure 7. Présentation d'un indicateur dans le KIK dédié à AGREGA [DOU 18].

\subsection{Grille de représentation : besoin de connaissance et pertinence des indicateurs}

La galerie «GKR » (Grille Kerbabel de Représentation) propose un processus de recensement et de collecte des différentes formes de connaissances qui peuvent être mobilisées par différents acteurs pour des démarches de représentation ou d'évaluation d'un système [DOU 15]. De plus, par ce processus, chaque acteur (notamment les scientifiques, experts, producteurs de granulats, l'IAU,...) a la possibilité de situer et de signifier la pertinence de ses connaissances par rapport au contexte. Les indicateurs pertinents pour ces démarches de représentation ou d'évaluation seront en effet resitués par rapport aux formes de connaissances qui fondent leur définition, ainsi que par rapport aux modes de production de ces connaissances : approches conceptuelles (scientifiques, vernaculaires ou autres), outils (d'analyse, de modélisation...), et pratiques diverses.

La Grille de représentation est conçue selon quatre axes. Elle donne à voir pour chaque indicateur (1) les catégories d'acteurs - appelés ici «porteurs de connaissances » - susceptibles de mobiliser cet indicateur; (2) les concepts, méthodes, outils ou théories employés pour l'utiliser; (3) les scénarios ou objets pour la comparaison desquels il peut être utilisé; (4) les enjeux qu'il permet d'aborder.

Les deux premiers axes donnent accès à la compréhension des sources mobilisées. Il s'agit en quelque sorte de l'Offre de connaissances, ce qui permet le recueil de formes de connaissances (concept d'indicateur, proto-indicateur, indicateurs...).

Les deux derniers axes structurent la contextualisation des connaissances en recensant les scénarios ou objets à comparer et les enjeux de soutenabilité. Ces deux axes représentent les Demandes de connaissances.

La GKR propose, enfin, d'évaluer la pertinence de la connaissance pour la situation d'évaluation, selon les quatre axes retenus [VAN 08] [DOU 09] [FUN 94]. Les porteurs de connaissances, mais aussi tout autre acteur intéressé, indiquent pour cela un coefficient de pertinence pour chacun des indicateurs retenus et pour chaque croisement entre les quatre axes qui auront été proposés. Chaque 
acteur devra choisir entre « $0 »$ qui signifie «Pas de pertinence » (valeur par défaut), « $1 »$ pour «Pertinence faible $»$, et « 4 » pour «Pertinence forte $»$ (voir figure 8 .).

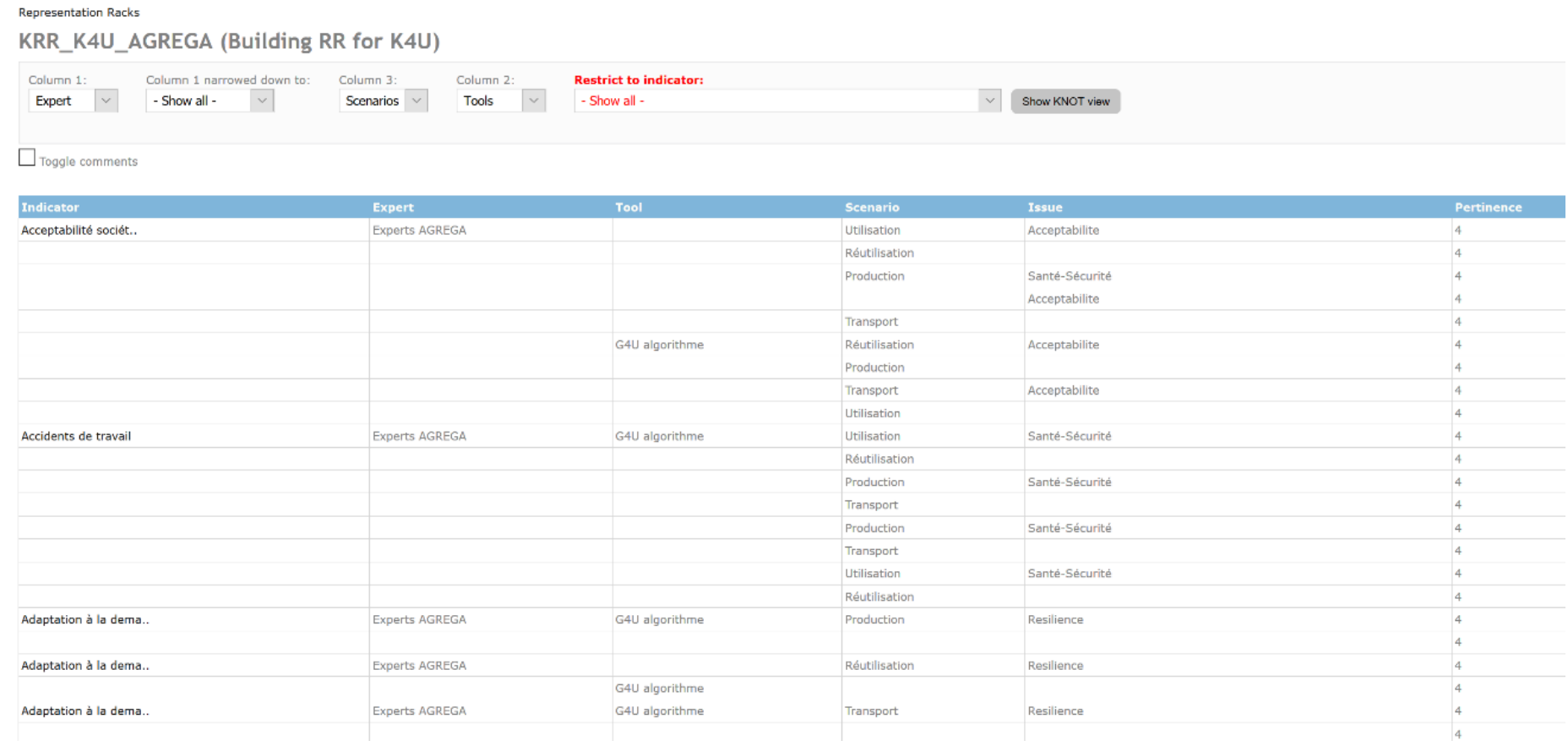

Figure 8. Indicateurs dans la grille de représentation [DOU 18].

Dans le cadre AGREGA, la GKR a été utilisée de manières ex-ante et ex-post, comme un complément aux sessions d'évaluation de 5 scénarios : Aménagements du territoire autour du Grand Paris Express; Grand Paris, une Métropole Durable ; Ouverture du Canal Seine-Nord Europe ; Développement de plateformes multimodales ; Construction du Grand Paris ; et «Zéro déchets ».

Le travail d'identification de la pertinence des indicateurs a conduit à proposer une liste d'indicateurs susceptibles d'être utilisés lors des évaluations.

\section{5. Évaluation multicritère de la performance grâce à l'outil K4U}

\subsection{Des enjeux de performances aux critères d'évaluation}

La galerie «K4U » (Kerbabel for You) du portail «ePLANETe.Blue » propose aux utilisateurs une méthode de coproduction d'algorithmes permettant d'évaluer la performance d'un système selon plusieurs critères de soutenabilité, par exemple le social ; l'écosystème ; l'économie ; la gouvernance; et la propagation des solutions soutenables. Ces critères de performance de soutenabilité sont précisés, chacun, par des objectifs subsidiaires.

Ces critères (et objectifs subsidiaires) de performance sont mis en regard (croisés) avec des objets analytiques qui contribuent à la performance du système (par exemple les différentes phases d'un cycle de vie). Des indicateurs de performance permettent d'évaluer comment un objet à comparer contribue à la performance du système. L'ensemble des indicateurs de performance, réunis en une formule algorithmique simple, permet d'évaluer les contributions respectives des différents objets à comparer à la réalisation des performances considérées, et ceci selon chacun des critères.

Le choix des indicateurs et la pondération des objectifs au sein de l'algorithme peut s'effectuer dans le cadre d'une négociation sociale, en ayant notamment recours aux méta-informations 
présentes dans le kiosque aux indicateurs, à la sélection d'indicateurs pertinents sur la Grille de représentation GKR, et à la matrice de délibération (qui sera présentée dans la section 6).

Ce mode de construction amène à considérer des critères de performance communs, mais aussi des objectifs et indicateurs spécifiques aux objets à comparer et auxquels les acteurs donneront un sens particulier, dépendant de leur métier, du lieu, du moment et de l'échelle d'évaluation.

\subsection{Choisir et pondérer les indicateurs d'évaluation des performances}

Les indicateurs de performance mobilisés par l'outil K4U peuvent être issus d'une présélection ex-ante, ou introduits spécifiquement, lorsque cela est nécessaire à l'évaluation du critère considéré.

Le fonctionnement de K4U implique que les évaluateurs attribuent à chaque indicateur une note de performance en suivant des règles d'interprétation et de normalisation élaborées préalablement et collectivement. Ces règles de normalisation permettent de rendre comparables entre elles les appréciations d'indicateurs se rapportant à ces différentes évaluations, quantitatives ou qualitatives, en les ramenant à une valuation sur une échelle de 0 à 10 grâce à l'utilisation de tables de Likert composées collectivement sur ePLANETe.

Après attribution d'une note à chacun des indicateurs, l'algorithme K4U associe une note à chaque axe de performance, afin de générer un profil à $n$ dimensions de performances - autant que de critères (voir figure 9). Par défaut l'algorithme effectue la moyenne pondérée des notes attribuées aux indicateurs associés au critère considéré.

\subsection{Application du K4U au cas des Granulats}

Un K4U a été élaboré en marge du projet AGREGA (G4U - «Granulates for You »). A chacun de ces 5 axes de performance ont été associés 6 objectifs subsidiaires de performance, et à chacun de ces objectifs ont été associés des indicateurs thématiques, quantitatifs ou qualitatifs. Ceux-ci ont été déclinés afin de décrire l'influence de chaque phase du cycle de vie des granulats (les objets à comparer; colonnes du tableau 1) sur la réalisation d'un objectif subsidiaire de performance d'ancrage territorial soutenable de la filière régionale d'approvisionnement en granulats (lignes du tableau 1).

On reflète ainsi notamment la différence de point de vue entre les acteurs intervenant à différentes phases du cycle de vie des granulats.

Pour certains objectifs, un unique indicateur permet d'aborder l'ensemble des quatre phases du cycle de vie, et les règles d'interprétation et de normalisation sont les mêmes pour chacune de ces phases. Pour d'autres objectifs, un même indicateur couvre les quatre phases, mais des règles de normalisation différentes sont proposées, du fait des spécificités des activités de ces différentes phases. Pour d'autres encore, si une même logique de définition des indicateurs est associée à un objectif pour toutes les phases, les indicateurs, ainsi que les règles d'interprétation et de normalisation associées, varient selon les phases.

\subsection{Principaux résultats}

Construire l'outil G4U en associant à chaque critère de performance (social, écosystème, économie, gouvernance et propagation) six objectifs subsidiaires dont l'atteinte est décrite par une ligne d'indicateurs, permet un dispositif maniable et synthétique (présenté tableau 1). 


\begin{tabular}{|c|c|c|c|c|c|}
\hline & & \multicolumn{4}{|c|}{ Indicateurs } \\
\hline & & & quantitatifs & qualitatifs & \\
\hline \multicolumn{2}{|c|}{ Critères de performance } & \multicolumn{4}{|c|}{ Phase du cycle de vie } \\
\hline Axes & Objectifs & Production & Transport & Utilisation & Recyclage \\
\hline \multirow{7}{*}{ Social } & Santé-sécurité & \multicolumn{4}{|c|}{ Accidents de travail } \\
\hline & Emploi & \multicolumn{4}{|c|}{ Effectifs } \\
\hline & Satisfaction au travail & \multicolumn{4}{|c|}{ Absentéisme } \\
\hline & Pénibilité au travail & \multicolumn{4}{|c|}{ Pénibilité } \\
\hline & Employabilité & \multicolumn{4}{|c|}{ Compétences } \\
\hline & Nuisances sonores & \multicolumn{4}{|c|}{ Niveau admissible } \\
\hline & Matières & $\begin{array}{l}\text { Addition au } \\
\text { stock }\end{array}$ & Trafic & $\begin{array}{c}\text { Addition au } \\
\text { stock }\end{array}$ & $\begin{array}{l}\text { Gestion du } \\
\text { stock }\end{array}$ \\
\hline & Eau & \multicolumn{4}{|c|}{ Empreinte eau bleue } \\
\hline & Energie & \multicolumn{4}{|c|}{ Intensité énergétique } \\
\hline Ecosystème & Climat & \multicolumn{4}{|c|}{ Emissions de GES } \\
\hline & Qualité de l'air & \multicolumn{4}{|c|}{ Emissions de PM10 } \\
\hline & $\begin{array}{l}\text { Engagement } \\
\text { environnemental }\end{array}$ & \multicolumn{4}{|c|}{ Niveau d'engagement } \\
\hline \multirow{6}{*}{ Economie } & Cohérence physique & $\begin{array}{c}\text { Disponibilité } \\
\text { prévisionnelle }\end{array}$ & $\begin{array}{c}\text { Continuité de } \\
\text { charge }\end{array}$ & $\begin{array}{l}\text { Utilisation } \\
\text { rationnelle }\end{array}$ & $\begin{array}{l}\text { Qualité des } \\
\text { ressources }\end{array}$ \\
\hline & Rentabilité & \multicolumn{4}{|c|}{ Interprétation de l'EBE } \\
\hline & Coûts de revient & \multicolumn{4}{|c|}{ Dépenses d'exploitation } \\
\hline & Investissement & \multicolumn{4}{|c|}{ Amortissement des actifs } \\
\hline & Résilience & \multicolumn{4}{|c|}{ Adaptation à la demande \& gestion flux } \\
\hline & Valeur locale & \multicolumn{4}{|c|}{ Création de valeur locale } \\
\hline \multirow{6}{*}{ Processus } & Implication & \multicolumn{4}{|c|}{ Implication des acteurs } \\
\hline & Compréhension & \multicolumn{4}{|c|}{ Culture partagée } \\
\hline & Expérience & \multicolumn{4}{|c|}{ Expérience de la solution } \\
\hline & Représentativité & \multicolumn{4}{|c|}{ Diversité d'influences } \\
\hline & Dialogue social & \multicolumn{4}{|c|}{ Réflexion interne } \\
\hline & Impulsion & \multicolumn{4}{|c|}{ Leadership } \\
\hline & Système technique & \multicolumn{4}{|c|}{ Stratégie technique et prospective } \\
\hline & $\begin{array}{l}\text { Perspectives } \\
\text { économiques }\end{array}$ & $\begin{array}{c}\text { Disponibilités } \\
\text { comparées }\end{array}$ & $\begin{array}{c}\text { Coûts } \\
\text { comparés }\end{array}$ & $\begin{array}{c}\text { Coûts } \\
\text { comparés }\end{array}$ & $\begin{array}{c}\text { Coûts } \\
\text { comparés }\end{array}$ \\
\hline Propagation & Compatibilité & & Compatibilité & ec la filière & \\
\hline & Acceptabilité & & Acceptabil & ociétale & \\
\hline & Réglementation & & Evolution $r$ & mentaire & \\
\hline & Modèles d'affaires & & Montages jurid & économiques & \\
\hline
\end{tabular}

Tableau 1. Indicateurs G4U [OCO 15].

Le fonctionnement de l'outil G4U a été testé sur la base d'un cas fictif. Le mode de présentation des résultats apparaît figure 9.

\begin{tabular}{|c|c|c|}
\hline Production & Utilisation & Recyclage \\
\hline \multicolumn{3}{|l|}{ - G4U algorithme - } \\
\hline \multicolumn{3}{|l|}{ Average all Objects to compare } \\
\hline SOCIAL & \multicolumn{2}{|c|}{8.5714285714286} \\
\hline ECOLOGIQUE & \multicolumn{2}{|c|}{5.1666666666667} \\
\hline ECONOMIQUE & \multicolumn{2}{|c|}{7.8333333333333} \\
\hline GOUVERNANCE & \multicolumn{2}{|l|}{5} \\
\hline PROPAGATION & \multicolumn{2}{|c|}{8.1428571428571} \\
\hline \multicolumn{3}{|l|}{ Assessment Diagram Production } \\
\hline SOCIAL & \multicolumn{2}{|l|}{7.5} \\
\hline ECOLOGIQUE & \multicolumn{2}{|c|}{5.1666666666667} \\
\hline ECONOMIQUE & \multicolumn{2}{|c|}{7.8333333333333} \\
\hline GOUVERNANCE & \multicolumn{2}{|l|}{5} \\
\hline PROPAGATION & \multicolumn{2}{|c|}{3.66666666666667} \\
\hline
\end{tabular}
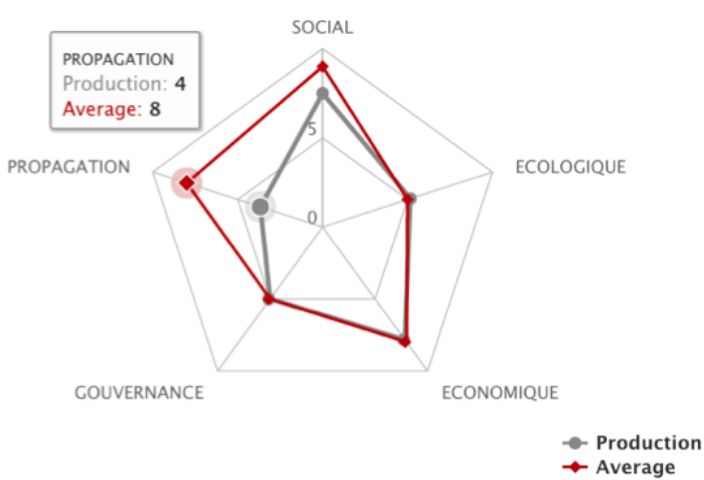

Figure 9. Résultats de l'analyse G4U sur le portail ePLANETe (cas fictif) 


\section{Innovation SPS : une construction de la signification sociale}

La Grille de Représentation et l'évaluation multicritère K4U permettent de rendre explicites la diversité des modes de représentation des acteurs impliqués dans les deux SPS orientés produit et usage, ainsi que les axes et les phases considérées pour l'analyse des performances de ces SPS. Il n'en demeure pas moins que face à un ensemble de scénarios, ces acteurs ont des appréhensions diverses concernant les risques encourus et les opportunités offertes.

L'optique retenue lors du projet AGREGA a été de comparer les différents scénarios du point de vue des onze enjeux de performance de soutenabilité grâce à la Matrice de Délibération [OCO 07]. Cette matrice fait à la fois référence à une méthode, et à un outil d'évaluation multi-acteurs et multicritères disponible en ligne sur le portail ePLANETe.Blue.

\subsection{Le défi d'une analyse multi-acteurs et multicritère: Les 3 axes}

Conçue sur l'idée du Rubik's Cube ${ }^{(\mathrm{TM})}$, la galerie Matrice Kerbabel de Délibération (MKD) permet de structurer selon trois axes (voir figure 10) la comparaison de formes d'injustices associées à différents conflits socio-environnementaux :

- Axe «Scénarios »-Quelles sont les situations à comparer ? Nous avons retenu les scénarios identifiés dans la Grille de Représentation ;

- Axe «Enjeux»-Quels sont les critères de comparaison ? Nous avons retenu les enjeux de performance identifiés dans la Grille de Représentation ;

- Axe «Acteurs»- Quelles sont les parties prenantes? Nous nous sommes référés aux acteurs identifiés lors du projet AGREGA.

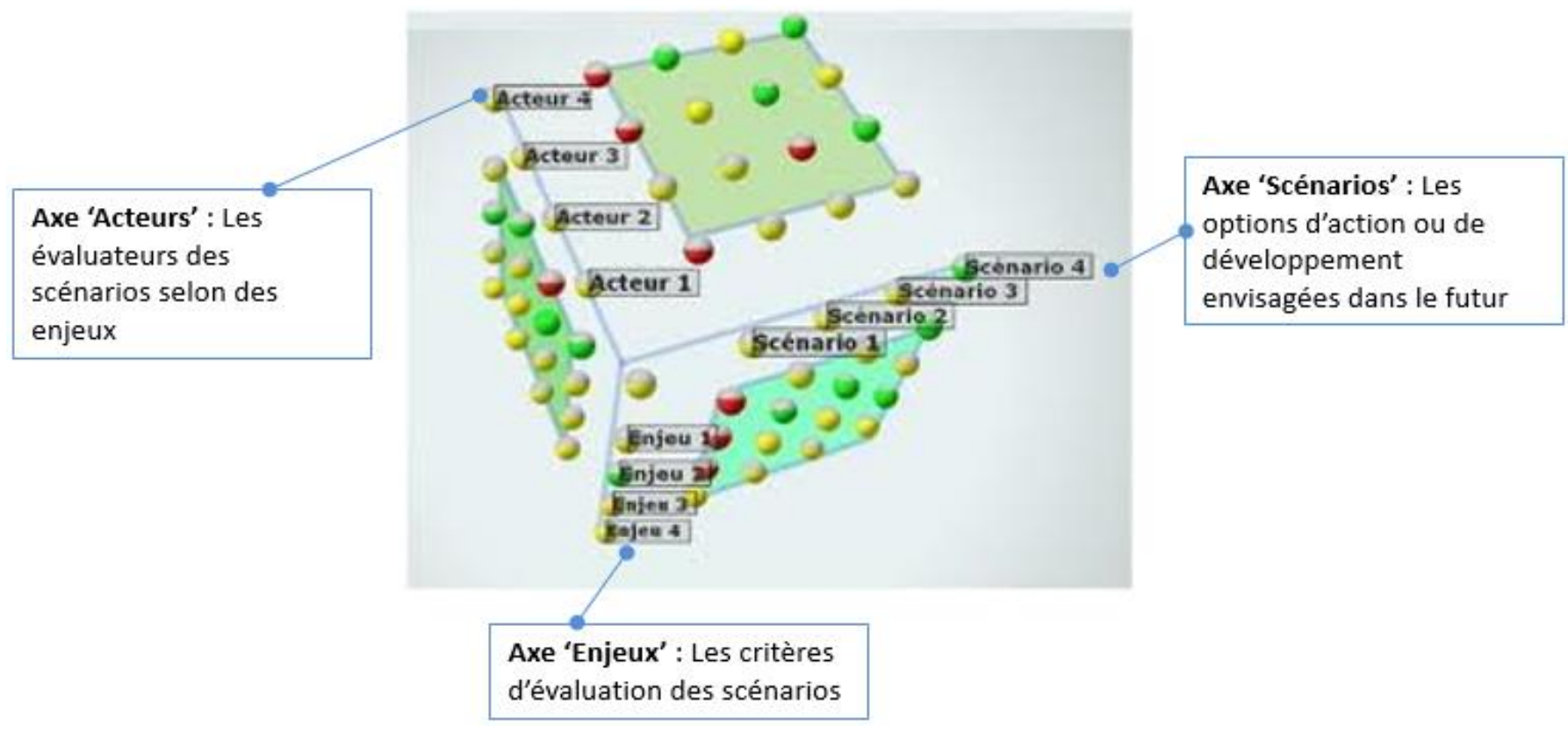

Figure 10. Résumé des axes impliqués dans l'évaluation subjective [DOU 18].

\subsection{La Matrice de Délibération: Trois niveaux d'analyse}

Après la définition des axes, il s'agit d'analyser, pour chacun des scénarios étudiés, les risques et opportunités, tels qu'exprimés par les acteurs, en les resituant par rapport aux enjeux de performance (soit 5 scénarios * 11 enjeux).

Un «panier » constitué d'1 à 5 indicateurs sera composé au niveau de chaque croisement entre les trois axes retenus (par exemple: quels indicateurs permettent de décrire le point de vue d'un 
transporteur de granulats sur l'enjeu de la «contribution au réchauffement climatique », dans le cadre du scénario «plateformes multimodales »?).

Ces indicateurs ont ici une vocation d'aide à la délibération. Ils peuvent être issus d'une présélection ex-ante ; c'est par exemple le cas lorsque l'on s'inspire des indicateurs situés sur la grille de représentation, ou d'entretiens d'évaluation au cours desquels les acteurs ont pu proposer des connaissances. Ils peuvent aussi être proposés par les acteurs s'ils estiment qu'un nouvel indicateur est nécessaire à l'expression d'un jugement concernant un croisement entre les 3 axes.

Plus généralement, il s'agit de sélectionner un petit nombre d'indicateurs qui, sans chercher l'exhaustivité, expriment la diversité des points de vue concernant le croisement des axes considérés, et serviront d'argument dans le jugement exprimé à travers ce panier d'indicateurs.

Pour chacun des indicateurs retenus, le jugement de valeur sera visualisé par une couleur : « vert » pour «Favorable »; « rouge » pour « Mauvais »; «jaune » pour «Incertain »; «blanc » pour «Ne sait pas»; «bleu » pour « Ne souhaite pas s'exprimer sur ce point ». Il est aussi possible de fournir un commentaire pour justifier le choix de couleur, et d'attribuer un poids subjectif (défini par l'acteur) pour pondérer l'importance de l'argument dans le jugement global concernant le croisement enjeu-scénario (voir tableau 2). Ici, ce n'est pas la quantification ou la qualification de l'indicateur qui importe, c'est le sens qu'il permet de fournir au jugement émis.

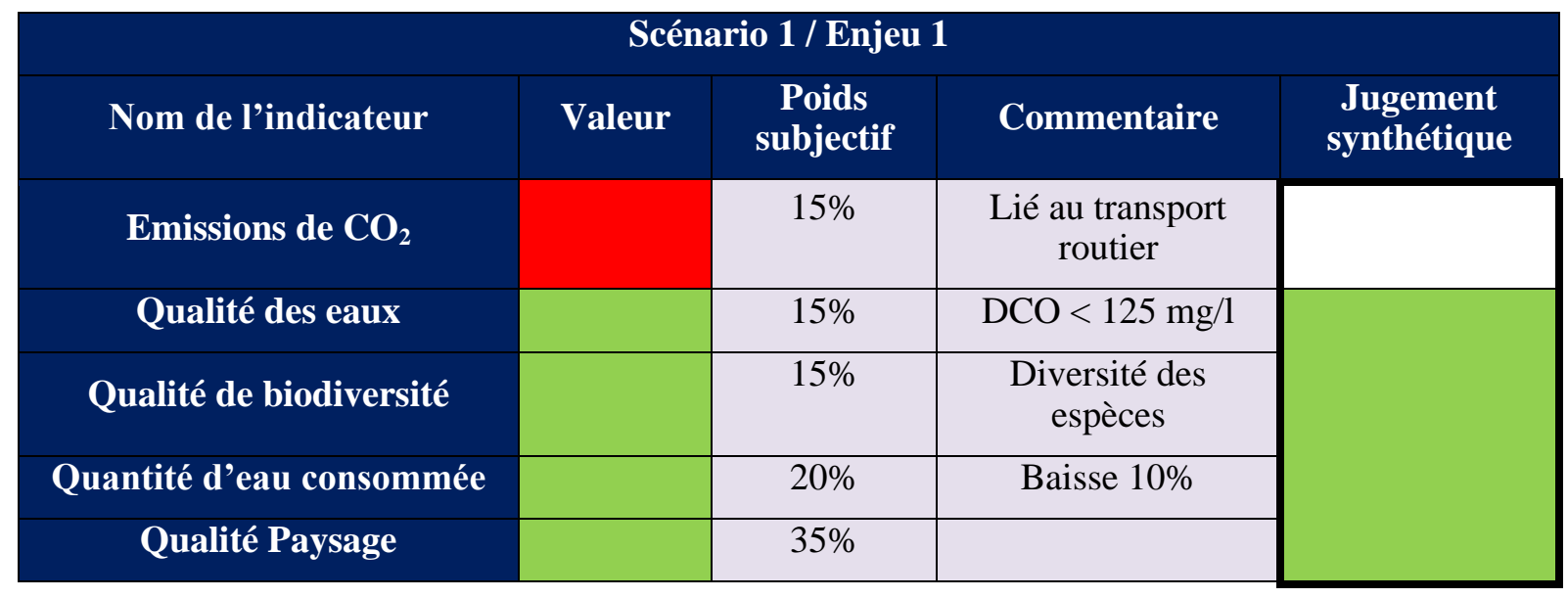

Tableau 2. Jugement composé dans la Matrice de Délibération par l'Acteur 1 concernant le croisement Scénario1 / Enjeu1 [DOU 18].

Lorsque que les valeurs retenues pour les indicateurs ne sont pas les mêmes, le jugement synthétique se remplit proportionnellement avec la couleur dominante (ici, au 4/5 avec VERT). Dans le cas d'une situation avec 2 indicateurs, l'un avec une valeur Verte et l'autre Rouge, c'est la valeur Rouge, la moins favorable, qui est retenue dans le jugement synthétique, cela favorise le dialogue entre les acteurs. Dans une situation avec 3 indicateurs et tous de valeur différente, c'est le Jaune, exprimant l'incertitude, qui apparaîtra comme jugement synthétique.

Pour une catégorie d'ACTEURS, les résultats de l'évaluation pour l'ensemble des scénarios et des enjeux se présentent, à ce premier niveau d'interprétation, sous la forme d'une « tranche » de la matrice (voir tableau 3). Chaque évaluation fournie par une catégorie d'acteurs est représentée par une « tranche ». 


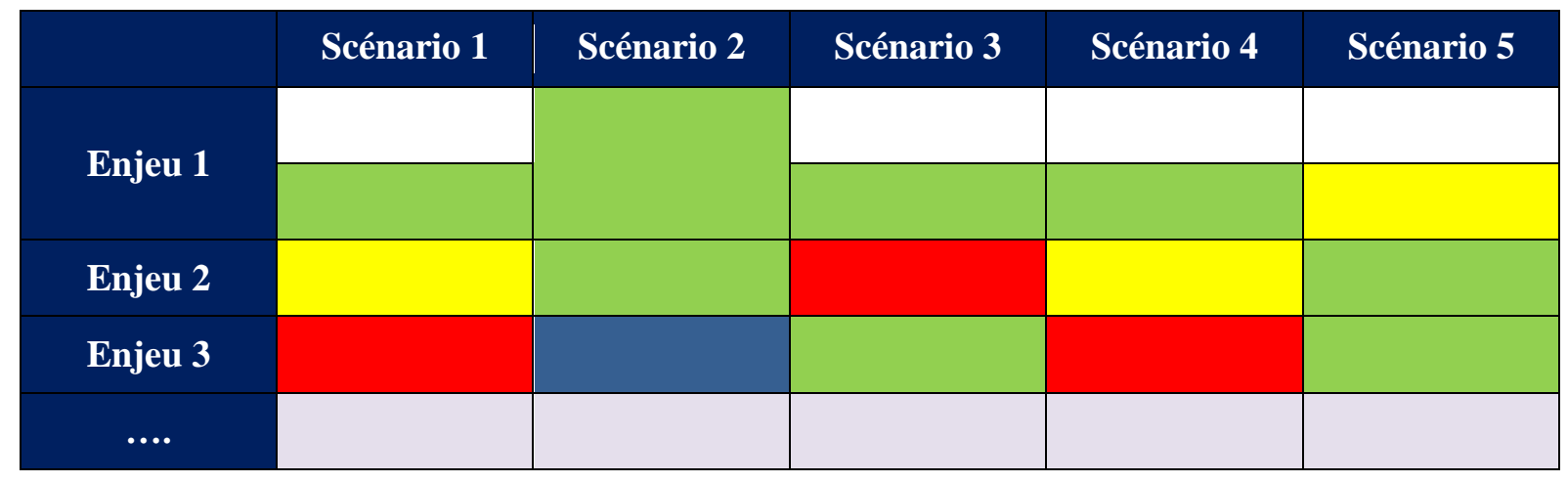

Tableau 3. Analyse des jugements, pour un acteur donné, dans la Matrice de Délibération [DOU 18].

Pour un SCENARIO, un deuxième niveau d'interprétation permet d'identifier une autre « tranche » correspondant cette fois-ci aux croisements ACTEUR/ENJEU (voir tableau 4).

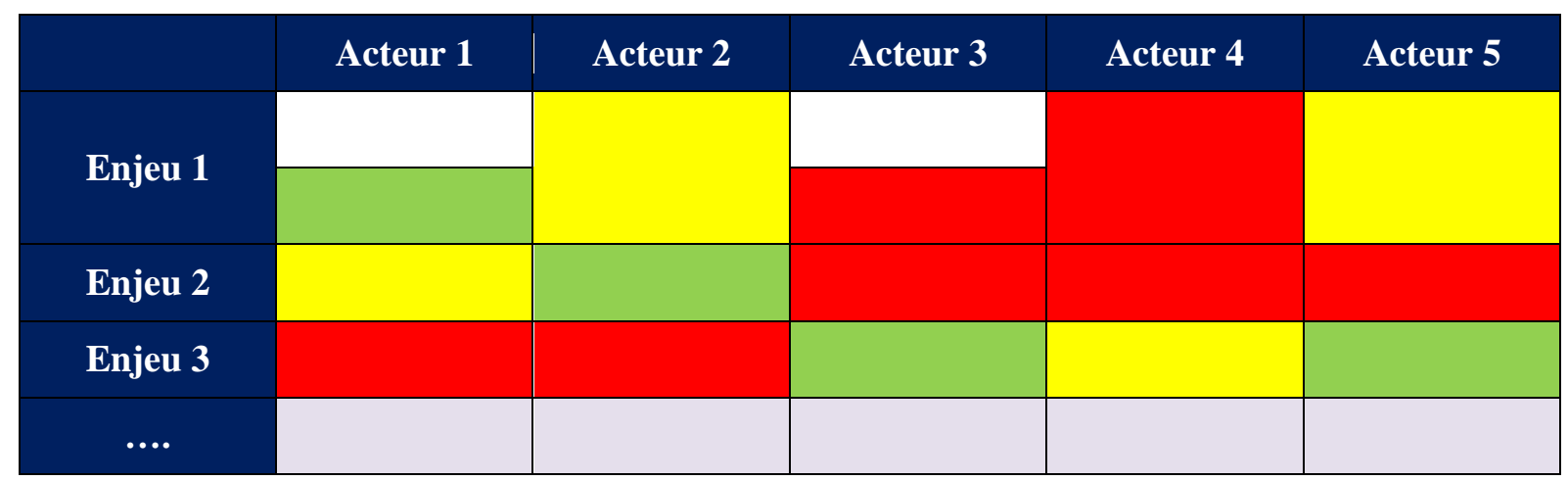

Tableau 4. Analyse des jugements portant sur les croisements Enjeux/Acteurs pour le Scénario 1, dans la Matrice de Délibération [DOU 18].

Un troisième niveau d'interprétation correspond à l'analyse des utilisations d'un indicateur donné dans la Matrice de Délibération afin d'identifier qui l'a mobilisé, pour parler de quel(s) enjeu(x), et pour quel(s) scénario(s) ? - (voir Tableau 5).

\subsection{Principaux résultats de la matrice AGREGA}

\subsubsection{Niveau des «tranches de la matrice »}

L'ensemble des tranches de la Matrice de Délibération a permis d'aborder les jugements portés par chaque catégorie d'acteurs sur le croisement entre chacun des 5 scénarios et des 11 enjeux considérés par le projet AGREGA (voir figure 11). 


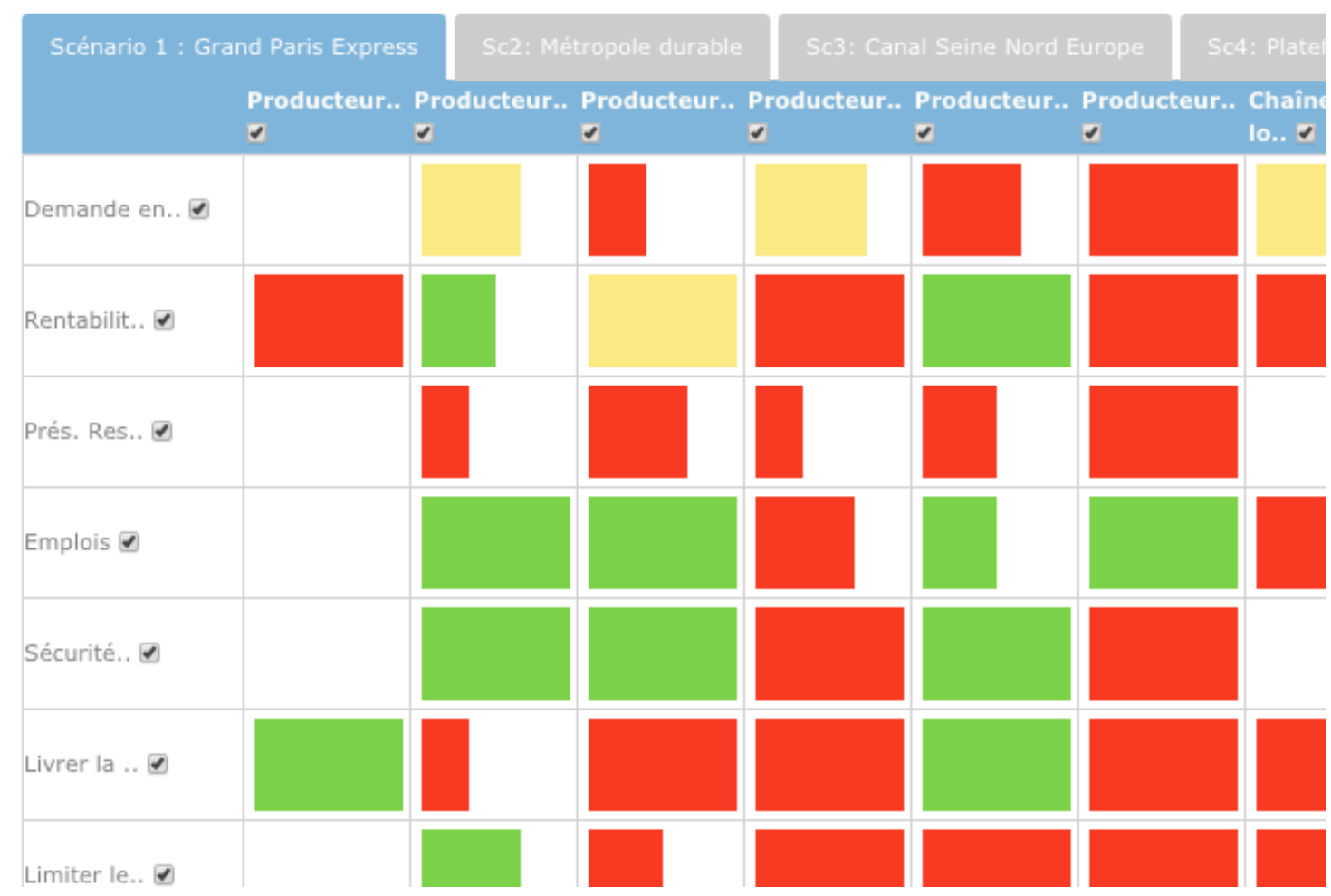

Figure 11. Vue partielle d'une tranche de la Matrice de Délibération [DOU 18].

\subsubsection{Niveau des «paniers d'indicateurs »}

Le panier d'indicateurs présenté figure 12 est composé des trois indicateurs (capacité de réponse, pérennité des activités et rupture d'approvisionnement) pondérés ici de la même manière (100\%).

Multicriteria Evaluations » Deliberation Matrix

\section{Deliberation Matrixes}

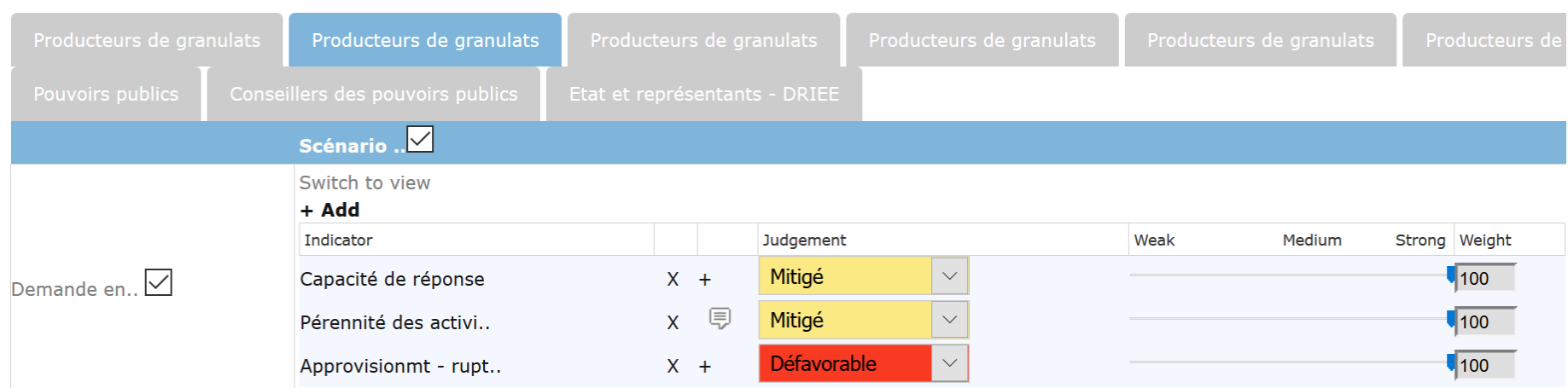

Figure 12. Panier d'indicateurs pour le croisement (Acteur : Producteur de granulats, Enjeu : Demande en Granulats, et Scénario 1 : Construire le Grand Paris) [DOU 18].

\subsubsection{Niveau des indicateurs}

Le tableau 5 offre une vision d'ensemble sur les jugements ayant mobilisé l'indicateur «émissions de $\mathrm{CO} 2$ », sur les acteurs ayant émis ces jugements, dans le cadre de quel scénario et à propos de quels enjeux. Le portail «ePLANETe.Blue» permet de générer directement ces informations selon cette structure. 


\begin{tabular}{|c|c|c|c|c|}
\hline Indicateur & $\begin{array}{l}\text { Jugement de } \\
\text { valeur }\end{array}$ & Catégorie d'acteur & Scénario considéré & $\begin{array}{l}\text { Enjeu } \\
\text { considéré }\end{array}$ \\
\hline \multirow{12}{*}{ CO2 émis } & $\begin{array}{l}\text { Défavorable } \\
(1 \mathrm{x})\end{array}$ & Utilisateurs de granulats $-\mathrm{BPE}$ & Sc2 : Métropole durable & $\begin{array}{l}\text { Contribution } \\
\text { Changement } \\
\text { Climatique }\end{array}$ \\
\hline & $\begin{array}{l}\text { Favorable } \\
(1 \mathrm{x})\end{array}$ & Utilisateurs de granulats - BPE & $\begin{array}{l}\text { Sc4 : Plateformes } \\
\text { multimodales }\end{array}$ & $\begin{array}{l}\text { Contribution } \\
\text { Changement } \\
\text { Climatique }\end{array}$ \\
\hline & Mitigé (1x) & Utilisateurs de granulats - BPE & Sc5 : «Zéro déchet » & $\begin{array}{l}\text { Contribution } \\
\text { Changement } \\
\text { Climatique }\end{array}$ \\
\hline & Mitigé (1x) & $\begin{array}{l}\text { Producteurs de granulats - } \\
\text { recyclés }\end{array}$ & Sc5 : «Zéro déchet» & $\begin{array}{l}\text { Contribution } \\
\text { Changement } \\
\text { Climatique }\end{array}$ \\
\hline & $\begin{array}{l}\text { Défavorable } \\
\text { (1x) }\end{array}$ & Producteurs de granulats & $\begin{array}{l}\text { Scénario } 1 \text { : Grand Paris } \\
\text { Express }\end{array}$ & $\begin{array}{l}\text { Livrer la } \\
\text { ressources }\end{array}$ \\
\hline & $\begin{array}{l}\text { Favorable } \\
(1 \mathrm{x})\end{array}$ & Producteurs de granulats & $\begin{array}{l}\text { Scénario } 1: \text { Grand Paris } \\
\text { Express }\end{array}$ & $\begin{array}{l}\text { Indépendance } \\
\text { IDF }\end{array}$ \\
\hline & $\begin{array}{l}\text { Favorable } \\
(1 \mathrm{x})\end{array}$ & Producteurs de granulats & $\begin{array}{l}\text { Scénario } 1 \text { : Grand Paris } \\
\text { Express }\end{array}$ & $\begin{array}{l}\text { Contribution } \\
\text { Changement } \\
\text { Climatique }\end{array}$ \\
\hline & $\begin{array}{l}\text { Défavorable } \\
(1 \mathrm{x})\end{array}$ & Producteurs de granulats & $\begin{array}{l}\text { Scénario } 1 \text { : Grand Paris } \\
\text { Express }\end{array}$ & $\begin{array}{l}\text { Contribution } \\
\text { Changement } \\
\text { Climatique }\end{array}$ \\
\hline & $\begin{array}{l}\text { Favorable } \\
(1 \mathrm{x})\end{array}$ & Etat et représentants - DRIEE & $\begin{array}{l}\text { Scénario } 1 \text { : Grand Paris } \\
\text { Express }\end{array}$ & $\begin{array}{l}\text { Contribution } \\
\text { Changement } \\
\text { Climatique }\end{array}$ \\
\hline & $\begin{array}{l}\text { Favorable } \\
(1 \mathrm{x})\end{array}$ & Etat et représentants - DRIEE & Sc2 : Métropole durable & $\begin{array}{l}\text { Contribution } \\
\text { Changement } \\
\text { Climatique }\end{array}$ \\
\hline & $\begin{array}{l}\text { Favorable } \\
(1 \mathrm{x})\end{array}$ & Etat et représentants - DRIEE & $\begin{array}{l}\text { Sc3 : Canal Seine Nord } \\
\text { Europe }\end{array}$ & $\begin{array}{l}\text { Contribution } \\
\text { Changement } \\
\text { Climatique }\end{array}$ \\
\hline & Mitigé (1x) & Producteurs de granulats & $\begin{array}{l}\text { Sc3 : Canal Seine Nord } \\
\text { Europe }\end{array}$ & $\begin{array}{l}\text { Contribution } \\
\text { Changement } \\
\text { Climatique }\end{array}$ \\
\hline Total & $\begin{array}{l}100 \% \\
\text { Défavorable } \\
(3 \mathrm{x}), 87 \% \\
\text { Favorable } \\
(6 \mathrm{x}), 100 \% \\
\text { Mitigé (3x) }\end{array}$ & $\begin{array}{l}\text { Utilisateurs de granulats }- \text { BPE } \\
(3 \mathrm{x}), \text { Producteurs de granulats - } \\
\text { recyclés }(1 \mathrm{x}), \text { Producteurs de } \\
\text { granulats }(3 \mathrm{x}), \text { Producteurs de } \\
\text { granulats }(1 \mathrm{x}), \text { Etat et } \\
\text { représentants }- \text { DRIEE }(3 \mathrm{x}), \\
\text { Producteurs de granulats }(1 \mathrm{x})\end{array}$ & $\begin{array}{l}\text { Sc2 }: \text { Métropole durable } \\
(2 \mathrm{x}), \operatorname{Sc} 4: \text { Plateformes } \\
\text { multimodales }(1 \mathrm{x}), \operatorname{Sc} 5: \\
\text { «Zéro déchet } ~(2 \mathrm{x}), \\
\text { Scénario } 1: \text { Grand Paris } \\
\text { Express }(5 \mathrm{x}), \operatorname{Sc} 3: \text { Canal } \\
\text { Seine Nord Europe }(2 \mathrm{x})\end{array}$ & $\begin{array}{l}\text { Contribution } \\
\text { Changement } \\
\text { Climatique } \\
(10 \mathrm{x}), \text { Livrer } \\
\text { la ressources } \\
(1 \mathrm{x}), \\
\text { Indépendance } \\
\text { IDF }(1 \mathrm{x})\end{array}$ \\
\hline
\end{tabular}

Tableau 5. Analyse de l'utilisation de l'indicateur $\mathrm{CO}_{2}$ [DOU 18].

\section{Discussion et retours d'expérience de cette démarche intégrée d'évaluation de la performance d'un SPS AGREGA.}

A travers cet exemple de mobilisation d'outils de la plateforme «ePLANETe.Blue », il s'agissait de présenter une démarche interdisciplinaire, au sens large, mobilisant des formes variées de connaissances et des méthodes ainsi que des outils conviviaux pour une diversité d'acteurs, afin de 
les accompagner dans des processus d'évaluation des scénarios d'approvisionnement en granulats de construction et de valorisation des déchets.

\subsection{Le défi de la gouvernance du travail collectif sur un portail web contributif}

Les acteurs n'ont pas tous la même compétence d'utilisation des outils participatifs en ligne tels que ceux proposés par la plateforme «ePLANETe.Blue». Il peut s'avérer utile dans certains cas d'effectuer les sessions d'évaluation en présentiel et sur support papier, puis de saisir les résultats sur le portail. C'est le choix qui a été fait dans le cadre du projet AGREGA. Une autre limite souvent évoquée est celle de la gouvernance de l'outil, de la neutralité de l'animateur ou du groupe animateur. Il n'y a à ce sujet pas d'ambiguïté : le travail web participatif implique de prendre des précautions similaires à celles qui doivent être envisagées dans les délibérations traditionnelles, afin notamment d'assurer la représentativité des points de vue.

Le web contributif est un support dont l'utilisation ne dispense pas d'une gouvernance sociale du travail collectif. Cependant, le principe même de fonctionnement du portail «ePLANETe.Blue » apporte des garanties que les délibérations traditionnelles ne confèrent pas. La conservation et la transparence des méta-informations associées aux indicateurs, les forums de discussion sur lesquels sont précisés les jugements de valeur émis par les utilisateurs, ainsi que la mise en situation des indicateurs par rapport aux méthodes d'analyses mobilisées par les uns et les autres, sont autant de caractéristiques qui facilitent la représentation partagée. La visibilité de cette représentation partagée a au moins deux effets : d'une part la responsabilisation de chacun dans l'expression de son point de vue (dont la trace subsiste de façon accessible à tous les participants), d'autre part l'augmentation de la compréhension réciproque favorisée par l'utilisation discursive des différentes galeries (approche «parcours de connaissance »).

\subsection{Le défi de l'évaluation de la performance d'un SPS dans l'optique d'une économie circulaire}

Augmenter l'ambition d'une stratégie SPS fondée sur une logique d'économie circulaire orientée produit, pour l'inclure dans une stratégie territoriale orientée usage ou résultat, implique de réfléchir à «quelles performances sont considérées » et à «qui est en mesure de les évaluer». Une autre question à aborder est celle des outils et exemples existants et disponibles qui permettent de les mesurer. La particularité de l'ouverture à de nouvelles dimensions de performance, lors du passage d'une ambition de SPS orienté produit à une ambition de SPS orienté usage, est que l'on se confronte à une analyse multi-échelle. Il est alors nécessaire d'analyser conjointement divers signaux, correspondant à une pluralité d'interprétations qualitatives des mêmes indicateurs décrivant la performance du système. Structurer la démarche de représentation du système et de ses performances autour d'un cas d'étude lié au secteur du granulat est intéressant car il devient possible de faire apparaître quatre phases du cycle de vie des granulats (production, transport, consommation, recyclage). On est alors conduit à intégrer la prise en compte des spécificités des activités et la question des échelles, ajoutant ainsi une dimension supplémentaire de signaux d'analyse des performances.

\subsection{Articulation de la méta-méthode INTEGRAAL et du portail web contributif ePLANETe.BIUe}

La méta-méthode INTEGRAAL structure cette démarche d'objectivation de multiples dimensions de performance de l'économie des granulats et accompagne la construction du sens donné à ces performances par des acteurs variés. Le portail «ePLANETe.Blue » est conçu pour faciliter cette double approche de représentation d'un système et de construction de la signification sociale des fonctionnalités, ce qui en fait un support utile lorsqu'il s'agit de penser à un couplage 
structurel entre un SPS orienté produit et un SPS orienté usage. L'analyse de la signification sociale est le préalable à l'acceptabilité d'une stratégie SPS orientée résultat caractérisée par un financement ex-ante - ou du moins par une impulsion partagée - des nouvelles conformations de l'économie des granulats.

\subsection{Complémentarité entre matrice de délibération et évaluation multicritère K4U}

La Matrice de délibération permet de construire la signification sociale des scénarios par rapport à des enjeux de performance issus d'entretiens menés avec des acteurs, donc par rapport à des enjeux au sens bien établi et ancré dans un contexte, mais auquel il est nécessaire de trouver des modes d'évaluation. L'évaluation multicritère $K 4 U$ propose des critères d'évaluation normative de ces performances, plus abstraits que les enjeux de performance exprimés par les acteurs, mais aux modalités d'évaluation bien définies.

Le cheminement discursif de l'un à l'autre de ces outils catalyse la médiation des connaissances sur le portail «ePLANETe.Blue », et préfigure en cela l'émergence potentielle de stratégies et de logiques de décision nouvelles, compatibles avec des innovations SPS.

\subsection{Perspectives}

$\mathrm{Au}$ niveau d'analyse portant sur la circularité de la matière, les contributeurs au portail ePLANETe. Blue pourraient produire un algorithme K4U local appuyé sur des indicateurs descriptifs des performances industrielles de l'économie du granulat.

Au niveau d'analyse portant sur la soutenabilité de l'inscription territoriale de la filière, d'autres contributeurs - ou en partie les mêmes - pourraient produire un algorithme K4U territorial qui décrirait les performances de soutenabilité de l'économie des granulats.

Sur une matrice de délibération, les acteurs impliqués dans ces deux analyses pourraient émettre des signaux qualitatifs concernant l'acceptabilité des contraintes imposées au système industriel par des choix territoriaux de politiques écologiques, économiques ou sociales, et réciproquement.

Les nouveaux indicateurs proposés lors de cette délibération pourraient entrer dans la composition d'un algorithme d'évaluation des performances de couplage structurel entre SPS orienté produit et SPS orienté usage, et préfigurer l'ambition partagée d'un SPS orienté résultat.

\section{Conclusion}

Le Portail de Connaissances «ePLANETe. Blue » s'inscrit dans le mouvement de création d'un web herméneutique permettant la découverte et la délibération des problématiques du développement soutenable. Il peut être considéré, d'une manière plus technique, comme une approche novatrice et expérimentale de la modélisation intégrative participative des systèmes écolosocio-économiques.

Ce portail a le potentiel d'accompagner de nouvelles approches de l'économie politique territoriale, de composer avec les contraintes usuelles de décision collective liées à la subjectivité des acteurs, à leur nombre, aux dimensions d'analyses et aux changements d'échelles. Il permet notamment la co-production d'une méthodologie d'évaluation ad hoc fondée sur la représentation partagée et la délibération, destinée à favoriser l'émergence de compromis locaux et circonstanciés entre acteurs ne mobilisant au départ pas les mêmes enjeux, espaces, temporalités et rationalités d'analyses. 


\section{Bibliographie}

[BAI 07] Baines T. S., Lightfoot H. W., Evans S., Neely A., Greenough R., Peppard J., ... \& Alcock J. R., « State-ofthe-art in product-service systems ». Proceedings of the Institution of Mechanical Engineers, Part B - Journal of engineering Manufacture, 221(10), 1543-1552, 2007.

[CHA 09] Chamaret A., Reichel V. \& O'CONnOR M., Les avenirs de la Boucle de Moisson. Un projet de réflexion participative pour un développement territorial soutenable. Rapport final, C3ED. Rambouillet. 118 p., UVSQ, 2009.

[CHA 15] CHAMARET A. (2015). Comprendre et structurer le système "Approvisionnement de granulats en Ile-deFrance" : ses acteurs, ses enjeux et les scénarios possibles - Synthèse des entretiens avec les acteurs. Tâche AGREGA n³.3. Rapport Adret et territoires pour Reeds - UVSQ.

[DOU 18] Douguet J-M., Morlat C., O’Connor M., Lanceleur, P., Evaluation multi-acteurs et multicritères des scénarios d'approvisionnement en granulats et d'évauation et de valorisation des déchets pour la construction du Grand Paris. Projet ANR 13-ECOT-0008: Anticipation et Gestion régionales des Ressources En Granulats (AGREGA), janvier 2018.

[DOU 15] Douguet J-M., O’CONNOR M., LANCELeur P., Eléments pour la création de la Grille Kerbabel ${ }^{\mathrm{TM}}$ de Représentation. Cahier de recherche Reeds - Université de Versailles-Saint-Quentin-en-Yvelines, 2015.

[DOU 09] Douguet J.M., O'CONNOR M. \& VAN DER SLUIJS J.P., «Tools to assess uncertainty in a deliberative perspective. A Catalogue. »Science for Policy: Opportunities and Challenges, Oxford University Press, India, 2009.

[FRA 11] FRAME B. \& O'CONNOR M., «Integrating valuation and deliberation: the purposes of sustainability assessment», Environmental Science \& Policy, 14(1), 1-10, 2011.

[FUN 94] FUNTOWICZ S.O., RAVETZ J. R. «The worth of a songbird: ecological economics as a post-normal science », Ecological Economics, 10, 197-207, 1994.

[GOE 99] Goedkoop M. J., Van Halen C. J., Te Riele H. R., \& Rommens P. J., «Product service systems, ecological and economic basics ». Report for Dutch Ministries of environment (VROM) and economic affairs (EZ), 36(1), 1-122, 1999.

[HOC 02] HocKERTS K., \& WEAVER N., « Towards a Theory of Sustainable Product Service Systems-What are the Dependent and Independent Variables of S-PSS », dans INSEAD-CMER Research Workshop, Sustainable Product Service Systems-Key Definitions and Concepts (Vol. 9), mai 2002.

[INS 15] INSEE (2015). Nomenclatures d'activités et de produits françaises NAF rév.2 - CPF rév.2.1.

[LAP 13] LAPERCHE B., \& PICARD F., «Environmental constraints, Product-Service Systems development and impacts on innovation management: learning from manufacturing firms in the French context ». Journal of Cleaner Production, 53, 118-128, 2013.

[LAU 08] LAURIOL J., «Développement durable et économie de la fonctionnalité: une stratégie renouvelée pour de nouveaux enjeux »,L'économie de la fonctionnalité une voie pour articuler dynamique économique et développement durable: enjeux et débats, 11-28., 2008.

[MAT 94] MATURANA H., \& VAREla F., L'arbre de la connaissance, Addison-Wesley France, Paris, 1994.

[MOR 06] MoReLLI N., « Developing new product service systems (PSS): methodologies and operational tools ». Journal of Cleaner Production, 14(17), 1495-1501, 2006.

[OCO 07] O'CONNOR M., Deliberative Sustainability Assessment with the on line kerDST Deliberation Support Tool. Cahier du C3ED N07-03, janvier 2007.

[OCO 11] O'CONNOR M., \& Da Cunha C., Présentation de la démarche INTEGRAAL pour le projet D2SOU. REEDS Resarch Reports, RRR N 2011-05, Technical Report, January 2011.

[OCO 15] O'Connor M., Douguet J. M., Morlat, C., Principes et fonctionnement de G4U, un outil d'évaluation multicritères des solutions d'anticipation et de gestion des ressources par la filière des granulats de construction de la région Ile-de-France. Rapport de Recherche REEDS, Rambouillet, REEDS - UVSQ, 2015.

[PAN 17] PANORAMAIDF, Granulats en Île-de-France : Panorama régional. Collectif DRIEE - IAU - UNICEM. 76 p, 2017. 
[PIP 16] PIPAME, Prospective: Marché actuel et offre de la filière minérale de construction et évaluation à échéance 2030 - Synthèse. Final report. DGE. 341 p., 2016.

[STA 86] STAHEL W.R., «The Functional Economy: Cultural and Organizational Change' », in 'Hidden innovation', Science \& Public Policy, London, Vol 13 No 4, August, 1986.

[TUK 04] TUKKERA., \& TISCHNER U., New business for old europe : product-service development as a means to enhance competitiveness and eco-efficiency; SusProNet (Sustainable Product-Service co-design Network) Union Européenne. Final report: 247 p., 2004.

[UNI 11] Union NATIONALE DES PRODUCTEURS DE GRANUlats, Carrières et granulats à l'horizon 2030, 22 propositions pour un approvisionnement durable des territoires. Livre Blanc. Union nationale des producteurs de granulats, 2011.

[VAI 09] VAILEANU-PAUN I., «La valeur entrepreneuriale comme construit institutionnel encastré dans le territoire dans le contexte de globalisation », dans Colloque Travail, capital savoir dans la mondialisation, Réseau de recherche sur l'Innovation, Grenoble, 2009.

[VAN 08] VAn Der Sluijs J.P., DOUguet J.-M., O’CONNOR M., Guimaraes Pereira A., Corral Quintana S. ET J.R. RAVETZ, "Qualité de la Connaissance dans un processus délibératif », Nature Science et Société 16, 265-273, EDP Sciences, DOI: 10.1051/nss:2008055, 2008. 